Department of Mathematics

Robust Boundary Conditions
for Stochastic Incompletely
Parabolic Systems of Equations

Markus Wahlsten and Jan Nordström

LiTH-MAT-R--2016/19--SE 
Department of Mathematics

Linköping University

S-581 83 Linköping 


\title{
Robust Boundary Conditions for Stochastic Incompletely Parabolic Systems of Equations
}

\author{
Markus Wahlsten $^{\mathrm{a}}$, Jan Nordström ${ }^{\mathrm{b}}$ \\ ${ }^{a}$ Department of Mathematics, Computational Mathematics, Linköping University, \\ SE-581 83 Linköping, Sweden (markus.wahlsten@liu.se). \\ ${ }^{b}$ Department of Mathematics, Computational Mathematics, Linköping University, \\ SE-581 83 Linköping, Sweden (jan.nordstrom@liu.se).
}

\begin{abstract}
We study an incompletely parabolic system in three space dimensions with stochastic boundary and initial data. We show how the variance of the solution can be manipulated by the boundary conditions, while keeping the mean value of the solution unaffected. Estimates of the variance of the solution is presented both analytically and numerically. We exemplify the technique by applying it to an incompletely parabolic model problem, as well as the one-dimensional compressible Navier-Stokes equations.

Keywords: uncertainty quantification, incompletely parabolic system, initial boundary value problems, stochastic data, variance reduction, robust design.
\end{abstract}

\section{Introduction}

The study of stochastic partial differential equations, with uncertainty in the boundary and initial data, is an important task in climatology [1], turbulent combustion [2], flow in porous media [3, 4], electromagnetics [5, 6] and seismology $[7,8]$ to name a few examples.

There are essentially two types of techniques that are used to quantify the uncertainty in the solution. Intrusive methods $[9,10,11,12,13,14]$ are based on polynomial chaos expansions, where the solution is expressed as a spectral expansion of the random variables. The method is cheap in terms of function evaluations for smooth problems, but requires a code, dealing specifically with the uncertainty. Non-intrusive methods are sample-based, i.e. the problem is solved several times for given values of the stochastic 
variables $[15,16,17,18]$. Quadrature techniques, often combined with sparse grid techniques $[19,20]$, are used to determine the statistical moments of the solution. The same code that deals with the deterministic case can be used.

The focus of this paper will be on how the choice of boundary conditions contributes to the size and distribution of the uncertainty in the solution. In [21], these effects were analyzed for hyperbolic problems and applied to the Euler and Maxwell's equations. In this paper we extend the analysis to include incompletely parabolic problems, such as the compressible NavierStokes equations. The analysis is valid both for intrusive and non-intrusive techniques.

In the first part of the paper, we analyze how the boundary conditions affect the stochastic properties of the solution by studying the energy rate of the solution. It is shown that the energy rate is equivalent to an evolution equation for the variance [21]. We proceed and study the possibility of reducing the uncertainty in the solution by choice of boundary conditions.

In the second part of the paper we make sure that the mathematical and numerical treatments of our initial boundary value problem is correct. Well-posed boundary conditions $[22,23,24,25,26]$, are derived. To discretize in space we use a finite difference scheme with Summation-by-Parts (SBP) operators $[27,28,29]$ and impose boundary conditions weakly by the Simultaneous-Approximation-Term (SAT) technique [30, 31, 32, 33]. We show that a convergent scheme is obtained. Quadrature rules based on the probability distributions are used to non-intrusively compute the statistical moments of the solution [34, 35, 36, 37].

In the third part of the paper, we exemplify the theoretical developments by numerical calculations. Numerical experiments are provided for a model problem and as a more realistic application, the compressible Navier-Stokes equations.

The rest of the paper will proceed as follows. In Section 2, the stochastic properties of the problem are analyzed, and estimates of the variance of the solution are derived. A model problem is studied in Section 4 where the implications of the technique are analyzed and discussed. In Section 5, the technique is applied to the one-dimensional Navier-Stokes equations. Finally, conclusions are drawn in Section 6. 


\section{The stochastic formulation}

Consider the following system of equations,

$$
\begin{aligned}
& u_{t}+A u_{x}+B u_{y}+C u_{z}=F_{x}(u)+G_{y}(u)+H_{z}(u), \quad \vec{x} \in \Omega, \quad t \geq 0, \\
& L u=g(\vec{x}, t, \vec{\xi}), \quad \vec{x} \in \partial \Omega, \quad t \geq 0, \\
& u=f(\vec{x}, \vec{\xi}), \quad \vec{x} \in \Omega, \quad t=0,
\end{aligned}
$$

where,

$$
\begin{aligned}
& F(u)=D_{11} u_{x}+D_{12} u_{y}+D_{13} u_{z}, \\
& G(u)=D_{21} u_{x}+D_{22} u_{y}+D_{23} u_{z}, \\
& H(u)=D_{31} u_{x}+D_{32} u_{y}+D_{33} u_{z}
\end{aligned}
$$

The solution is represented by the vector $u=u(\vec{x}, t, \vec{\xi})=\left[u_{1}, \ldots, u_{M}\right]$, where, $\vec{x}=(x, y, z)$, and $\vec{\xi}=\left(\xi_{1}, \xi_{2}, \ldots, \xi_{L}\right)$ is the vector of variables describing the stochastic variation of the problem. The $M \times M$ matrices $A, B, C, D_{i j}$ are constant and symmetric. The matrices $D_{i j}$ 's are singular, leading to an incompletely parabolic problem. $L$ is the boundary operator defined on the boundary $\partial \Omega . f(\vec{x}, \vec{\xi})$ and $g(\vec{x}, t, \vec{\xi})$ are the stochastic initial and boundary data to the problem. With a proper choice of the matrices involved, the problem (1) represents the linearized and symmetrized compressible NavierStokes equations [38].

In the following form of (1), we highlight the stochastic nature of the data,

$$
\begin{aligned}
u_{t}+A u_{x}+B u_{y}+C u_{z} & =F_{x}(u)+G_{y}(u)+H_{z}(u), \\
L u & =g(\vec{x}, t, \vec{\xi})=\mathbb{E}[g]+\delta g(\vec{x}, t, \vec{\xi}), \\
u & =f(\vec{x}, \vec{\xi})=\mathbb{E}[f]+\delta f(\vec{x}, \vec{\xi}) .
\end{aligned}
$$

The expected value is denoted by $\mathbb{E}[\cdot]=\mathbb{E}[\vec{x}, t]$ and the deviation from the mean is indicated by $\delta$. By taking the expected value of (3) and defining $v=\mathbb{E}[u]$ we obtain,

$$
\begin{aligned}
v_{t}+A v_{x}+B v_{y}+C v_{z} & =F_{x}(v)+G_{y}(v)+H_{z}(v), \\
L v & =\mathbb{E}[g] \\
v & =\mathbb{E}[f]
\end{aligned}
$$

where we have used that $F_{x}, G_{y}$ and $H_{z}$ are linear in $u$.

Remark 1. Equation (4) is equivalent to the equation determining the first term in a Polynomial Chaos expansion of u, see [39]. 
Next, the difference between (3) and (4) together with the notation $e=$ $u-v$ gives,

$$
\begin{aligned}
e_{t}+A e_{x}+B e_{y}+C e_{z} & =F_{x}(e)+G_{y}(e)+H_{z}(e), \\
L e & =\delta g(\vec{x}, t, \vec{\xi}) \\
e & =\delta f(\vec{x}, \vec{\xi}) .
\end{aligned}
$$

\subsection{Variance formulation}

The boundary conditions related to (5) will be provided on the form

$$
E^{-}-R E^{+}=\delta g,
$$

where

$$
E^{+}=\left[\begin{array}{c}
\tilde{e}^{+}-D^{+} \nabla \tilde{e} \\
Z_{+}^{T} \nabla \tilde{e}
\end{array}\right], \quad E^{-}=\left[\begin{array}{c}
\tilde{e}^{-}-D^{-} \nabla \tilde{e} \\
Z_{-}^{T} \nabla \tilde{e}
\end{array}\right],
$$

together with $\tilde{e}=X^{T} e, \tilde{e}^{-}=X_{-}^{T} e, \tilde{e}^{+}=X_{+}^{T} e$ and $\nabla \tilde{e}=\left[\tilde{e}_{x}, \tilde{e}_{y}, \tilde{e}_{z}\right]^{T}$. The matrices $X^{+}$and $X^{-}$are the eigenvectors corresponding to the positive and negative eigenvalues $\Lambda^{+}$and $\Lambda^{-}$of $(A, B, C) \cdot \vec{n}$, respectively. We denote $D^{ \pm}$ as

$$
D^{ \pm}=\left(\Lambda^{ \pm}\right)^{-1} \tilde{D}^{ \pm}, \quad \text { where } \tilde{D}=\left[\begin{array}{c}
\tilde{D}^{+} \\
\tilde{D}^{-}
\end{array}\right]
$$

The eigenvectors $Z_{ \pm}$correspond to the positive and negative eigenvalues of the matrix $-\tilde{D}^{T} \Lambda^{-1} \tilde{D}$. For a detailed derivation and further definitions, the reader is referred to Appendix A.

Next, we multiply (5) by $e$ and integrate in space. This procedure together with the use of (6) leads to the following equation,

$$
\|e\|_{t}^{2}+2 D I_{e}=-\oint_{\delta \Omega}\left[\begin{array}{c}
E^{+} \\
\delta g
\end{array}\right]^{T}\left[\begin{array}{cc}
R^{T} \Lambda_{\mathbf{M}}^{-} R+\Lambda_{\mathbf{M}}^{+} & \left(\Lambda_{\mathbf{M}}^{-} R\right)^{T} \\
\Lambda_{\mathbf{M}}^{-} R & \Lambda_{\mathbf{M}}^{-}
\end{array}\right]\left[\begin{array}{c}
E^{+} \\
\delta g
\end{array}\right] d s,
$$

where,

$$
D I_{e}=\int_{\Omega}\left[\begin{array}{l}
e_{x} \\
e_{y} \\
e_{z}
\end{array}\right]^{T}\left[\begin{array}{lll}
D_{11} & D_{12} & D_{13} \\
D_{21} & D_{22} & D_{23} \\
D_{31} & D_{32} & D_{33}
\end{array}\right]\left[\begin{array}{l}
e_{x} \\
e_{y} \\
e_{z}
\end{array}\right] d \Omega \geq 0,
$$

is the dissipation.

To simplify (8), we substitute some of the terms involving $\delta g$ using (6), and average the result with (8) to obtain

$$
\begin{aligned}
\|e\|_{t}^{2}+2 D I_{e}= & -\oint_{\delta \Omega}\left[\left(E^{+}\right)^{T} \Lambda_{\mathbf{M}}^{+}\left(E^{+}\right)+(\delta g)^{T} \Lambda_{\mathbf{M}}^{-}(\delta g)\right. \\
& \left.+\left(\delta g+E^{-}\right)^{T} \Lambda_{\mathbf{M}}^{-}\left(R E^{+}\right)\right] d s .
\end{aligned}
$$


Furthermore, we make the crucial observation that

$$
\mathbb{E}\left[\|e\|^{2}\right]=\mathbb{E}\left[\|u-\mathbb{E}[u]\|^{2}\right]=\int_{\Omega} \mathbb{E}\left[(u-\mathbb{E}[u])^{2}\right] d x d y d z=\|\operatorname{Var}[u]\|_{1} .
$$

By taking the expected value of (9) we obtain,

$$
\begin{aligned}
\frac{d}{d t}\|\operatorname{Var}[u]\|_{1}+2 \mathbb{E}\left[D I_{e}\right]= & -\oint_{\delta \Omega}\left[\mathbb{E}\left[\left(E^{+}\right)^{T} \Lambda_{\mathbf{M}}^{+}\left(E^{+}\right)\right]+\mathbb{E}\left[(\delta g)^{T} \Lambda_{\mathbf{M}}^{-}(\delta g)\right]\right. \\
& \left.+\mathbb{E}\left[\left(\delta g+E^{-}\right)^{T} \Lambda_{\mathbf{M}}^{-}\left(R E^{+}\right)\right]\right] d s .
\end{aligned}
$$

Note that since $D I_{e}$ is positive semi-definite we have, $2 \mathbb{E}\left[D I_{e}\right] \geq 0$.

To get a clear view of how the matrix $R$ influences the variance, we also take into account that the data depends on $R$, that is

$$
E^{-}-R E^{+}=\delta g=\delta g^{-}-R \delta g^{+}
$$

By using (12) in (9) we obtain the final result,

$$
\begin{aligned}
\frac{d}{d t}\|\operatorname{Var}[u]\|_{1}+2 \mathbb{E}\left[D I_{e}\right] & =-\oint_{\delta \Omega}\left[\mathbb{E}\left[\left(E^{+}\right)^{T} \Lambda_{\mathbf{M}}^{+}\left(E^{+}\right)\right]+\mathbb{E}\left[\left(\delta g^{-}\right)^{T} \Lambda_{\mathbf{M}}^{-}\left(\delta g^{-}\right)\right]\right. \\
& +\mathbb{E}\left[\left(R \delta g^{+}\right)^{T} \Lambda_{\mathbf{M}}^{-}\left(R \delta g^{+}\right)\right]-2 \mathbb{E}\left[\left(R \delta g^{+}\right)^{T} \Lambda_{\mathbf{M}}^{-}\left(\delta g^{-}\right)\right] \\
& \left.+\mathbb{E}\left[\left(\delta g^{-}-R \delta g^{+}+E^{-}\right)^{T} \Lambda_{\mathbf{M}}^{-}\left(R E^{+}\right)\right]\right] d s .
\end{aligned}
$$

Remark 2. Note that (13) is a complete description of how the variance develops in time, and how it depends on the boundary condition through $R$.

\subsection{The mean value}

Our ambition is to manipulate the variance in the problem without changing the mean. To that end, consider the two well-posed problems

$$
\begin{aligned}
u_{t}+P(u) & =F, \\
L_{1} u & =L_{1} w+\delta g_{u}, \\
u(\vec{x}, 0) & =w(\vec{x}, 0), \\
v_{t}+P(v) & =F, \\
L_{2} v & =L_{2} w+\delta g_{v}, \\
v(\vec{x}, 0) & =w(\vec{x}, 0),
\end{aligned}
$$

where $P(\cdot)$ denotes a general linear differential operator and $L_{1}, L_{2}$ are two different linear boundary operators. Moreover, $F$ is a deterministic forcing 
function, $w=w(\vec{x}, t)$ is a solution to the Cauchy problem [40] corresponding to the deterministic problems (14) and (15). Furthermore, $\delta g_{u}=\delta g_{u}(\vec{x}, t, \vec{\xi})$ and $\delta g_{v}=\delta g_{v}(\vec{x}, t, \vec{\xi})$ are added randomness. The fact that $w$ is a solution to the Cauchy problem implies that it satisfies the relation $w_{t}+P(w)=F$, in the whole space.

We need

Proposition 1. The expected value of the solutions to the two problems (14) and (15) are identical if $\delta g_{u}$ and $\delta g_{v}$ have zero mean.

Proof. We start by taking the expected value of (14) and (15), which yields

$$
\begin{aligned}
\bar{u}_{t}+P(\bar{u}) & =F, \\
L_{1} \bar{u} & =L_{1} w, \\
\bar{u}(\vec{x}, 0) & =w(\vec{x}, 0), \\
\bar{v}_{t}+P(\bar{v}) & =F, \\
L_{2} \bar{v} & =L_{2} w, \\
\bar{v}(\vec{x}, 0) & =w(\vec{x}, 0) .
\end{aligned}
$$

By taking the difference between (16) and (17) we find

$$
\begin{aligned}
\bar{e}_{t}+P(\bar{e}) & =0, \\
L_{1} \bar{u}-L_{2} \bar{v} & =L_{1} w-L_{2} w, \\
\bar{e}(\vec{x}, 0) & =0,
\end{aligned}
$$

where $\bar{e}=\bar{u}-\bar{v}$ represents the difference between the mean values.

Next, we rearrange the boundary conditions (substitute $\bar{u}$ with $\bar{e}+\bar{v}$ ) and obtain,

$$
\begin{aligned}
\bar{e}_{t}+P(\bar{e}) & =0, \\
L_{1} \bar{e} & =\tilde{L}(w-\bar{v}), \\
\bar{e}(\vec{x}, 0) & =0 .
\end{aligned}
$$

In (19), $\tilde{L}=L_{1}-L_{2}$. The result $\bar{e}=0$ follows from well-posedness if $w=\bar{v}$ on the boundary, which is true since $w$ solves (17).

Remark 3. Proposition 1 implies that we can focus on minimizing the variance. The expected value is unchanged for different well-posed boundary conditions provided that the data is given by an exact deterministic solution to the Cauchy problem with zero mean uncertainty added on. This situation is common in practice, where the boundary and initial data are given by wellknown geometrical and physical considerations together with uncertainties in the form of small stochastic perturbations. 


\subsection{Evaluation of variance}

We will consider the evaluation of the variance in (13) for three different scenarios; zero, decaying and large variance on the boundary.

\subsubsection{Zero variance on the boundary}

By assuming no uncertainty in the boundary data $\left(\delta g=\delta g^{+}=\delta g^{-}=0\right)$, (13) yields,

$$
\|\operatorname{Var}[u]\|_{t}+2 \mathbb{E}\left[D I_{e}\right]=-\oint_{\delta \Omega} \mathbb{E}\left[\left(E^{+}\right)^{T}\left(\Lambda_{\mathbf{M}}^{+}+R^{T} \Lambda_{\mathbf{M}}^{-} R\right)\left(E^{+}\right)\right] d s .
$$

In this case, the uncertainties in the problem are provided by the initial data. From (20) we clearly see that the optimal (in terms of variance reduction) choice of $R$ is zero and that all non-zero elements in $R$ will lead to an increase in the $L_{1}$-norm of the variance of the solution.

\subsubsection{Decaying variance on the boundary}

We now assume time-decaying variances in the boundary data. By considering only the terms depending on the matrix $R$ in (13), we get,

$$
-\oint_{\delta \Omega} \mathbb{E}\left(\left[\begin{array}{c}
E^{+} \\
E^{-} \\
\delta g^{+} \\
\delta g^{-}
\end{array}\right]^{T}\left[\begin{array}{cccc}
0 & 0 & 0 & 0 \\
\Lambda_{M}^{-} R & 0 & 0 & 0 \\
R^{T} \Lambda_{M}^{-} R & 0 & R^{T} \Lambda_{M}^{-} R & R^{T} \Lambda_{M}^{-} \\
-\Lambda_{M}^{-} R & 0 & \Lambda_{M}^{-} R & 0
\end{array}\right]\left[\begin{array}{c}
E^{+} \\
E^{-} \\
\delta g^{+} \\
\delta g^{-}
\end{array}\right]\right) d s .
$$

As can be seen in (21) the optimal choice of $R$ depends on the various correlations between the data $g^{+}, g^{-}$, and the variables $E^{+}$and $E^{-}$. However, in the long run, the problem is well-approximated by the zero variance on the boundary case. The optimal choice also in this case is $R=0$.

\subsubsection{Large variance on the boundary}

For large non-decaying uncertainty on the boundary, we cannot draw any general conclusions of the best choice of $R$ since the correlation terms in (21) will dominate. We will investigate this case numerically in Section 4.

\section{A stable and provably convergent approximation}

As a quality control for the upcoming numerical calculations, we derive well-posed boundary conditions and a provably convergent numerical approximation. The details are for ease of reading placed in Appendix A and Appendix B. The main theoretical results are given here for completeness. 


\subsection{Well-posedness}

Regarding well-posedness we have proved the following propositions

Proposition 2. The problem (1) with the homogeneous boundary conditions

$$
W^{-}-R W^{+}=0
$$

subject to

$$
R^{T} \Lambda_{\mathbf{M}}^{-} R+\Lambda_{\mathbf{M}}^{+} \geq 0
$$

is well-posed. In (22) and (23), we denote $W^{+}, W^{-}, \Lambda_{\mathbf{M}}^{-}$and $\Lambda_{\mathbf{M}}^{+}$by

$$
\begin{aligned}
& W^{+}=\left[\begin{array}{c}
w^{+}-D^{+} \nabla w \\
Z_{+}^{T} \nabla w
\end{array}\right], \quad \Lambda_{\mathbf{M}}^{+}=\left[\begin{array}{cc}
\Lambda^{+} & 0 \\
0 & \Lambda_{K}^{+}
\end{array}\right], \\
& W^{-}=\left[\begin{array}{c}
w^{-}-D^{-} \nabla w \\
Z_{-}^{T} \nabla w
\end{array}\right], \quad \Lambda_{\mathbf{M}}^{-}=\left[\begin{array}{cc}
\Lambda^{-} & 0 \\
0 & \Lambda_{K}^{-}
\end{array}\right] .
\end{aligned}
$$

with $w=X^{T} u, w^{+}=X_{+}^{T} u, w^{-}=X_{-}^{T} u$, and $\nabla w=\left[w_{x}, w_{y}, w_{z}\right]^{T}$, similarly to (7). The boundary conditions (22) relates to the boundary operator $L$ in (1) in the following way

$$
L u=W^{-}-R W^{+}, \quad \text { and } \quad W=\left[\begin{array}{l}
W^{+} \\
W^{-}
\end{array}\right]
$$

Proof. See Appendix A

Proposition 3. The problem (1) with non-homogeneous boundary conditions

$$
W^{-}-R W^{+}=g
$$

and the condition

$$
R^{T} \Lambda_{\mathbf{M}}^{-} R+\Lambda_{\mathbf{M}}^{+}>0
$$

is strongly well-posed.

Proof. see Appendix A 


\subsection{The numerical scheme}

The semi-discrete finite difference scheme we have used is based on the SBP-SAT formulation $[41,42,43,44]$. To shorten the rather lengthy analysis, we refer to [45] for technical details.

The semi-discrete version of (1) on SBP-SAT form can be written as,

$$
\begin{aligned}
v_{t} & +\left(D_{x} \otimes I_{y} \otimes I_{z} \otimes A\right) v+\left(I_{x} \otimes D_{y} \otimes I_{z} \otimes B\right) v \\
& +\left(I_{x} \otimes I_{y} \otimes D_{z} \otimes C\right) v \\
& =\left(D_{x} \otimes I_{y} \otimes I_{z} \otimes I_{M}\right) F+\left(I_{x} \otimes D_{y} \otimes I_{z} \otimes I_{M}\right) G \\
& +\left(I_{x} \otimes I_{y} \otimes D_{z} \otimes I_{M}\right) H \\
& +\left(P_{x}^{-1} E_{N_{x}} \otimes I_{y} \otimes I_{z} \otimes I_{M}\right)\left(\tilde{\Sigma}\left(\tilde{L}^{-}-\tilde{R} \tilde{L}^{+}\right) v-e_{N_{x}} \otimes g\right) \\
v(0) & =f .
\end{aligned}
$$

For simplicity, we have only included the boundary condition at $x=1$. The treatment of the remaining boundaries is completely analogous.

The discrete solution is arranged as

$$
v=\left[\begin{array}{c}
v_{0} \\
v_{1} \\
\vdots \\
{\left[v_{i}\right]} \\
\vdots \\
v_{N_{x}}
\end{array}\right], \quad\left[v_{i}\right]=\left[\begin{array}{c}
v_{0} \\
v_{1} \\
\vdots \\
{\left[v_{j}\right]} \\
\vdots \\
v_{N_{y}}
\end{array}\right]_{i} \quad\left[v_{j}\right]_{i}=\left[\begin{array}{c}
v_{0} \\
v_{1} \\
\vdots \\
{\left[v_{k}\right]} \\
\vdots \\
v_{N_{z}}
\end{array}\right]_{i j} \quad\left[v_{k}\right]_{j i}=\left[\begin{array}{c}
v_{0} \\
v_{1} \\
\vdots \\
v_{l} \\
\vdots \\
v_{M}
\end{array}\right]_{i j k}
$$

where $v_{i j k l}$ approximates $u_{l}\left(x_{i}, y_{j}, z_{k}, t, \xi\right)$.

In (28), $D_{x, y, z}=P_{x, y, z}^{-1} Q_{x, y, z}$ is the discrete approximation of the first derivative in space in the $x, y$ and $z$ direction, respectively. Moreover, $I_{x}$, $I_{y}, I_{z}$ and $I_{M}$ are the identity matrices of size $N_{x}+1, N_{y}+1, N_{z}+1$ and $M$. The matrices $E_{0_{x}}$ and $E_{N_{x}}$ are zero except for the element $(1,1)$ and $\left(N_{x}+1, N_{x}+1\right)$ respectively, which is equal to 1 . Similarly, the vector elements in $e_{N_{x}}$ are zero with the exception of the last element which is $1 . P_{x, y, z}$ is a positive definite diagonal matrix and $Q_{x, y, z}$ is an almost skew-symmetric matrix satisfying $Q_{x, y, z}+Q_{x, y, z}^{T}=E_{N_{x, y, z}}-E_{0 x, y, z}=\mathbb{B}=\operatorname{diag}[-1,0, \ldots, 0,1]$.

We have also introduced the numerical fluxes

$$
\begin{aligned}
& F=\left(\tilde{I} \otimes D_{11}\right) v_{x}+\left(\tilde{I} \otimes D_{12}\right) v_{y}+\left(\tilde{I} \otimes D_{13}\right) v_{z}, \\
& G=\left(\tilde{I} \otimes D_{21}\right) v_{x}+\left(\tilde{I} \otimes D_{22}\right) v_{y}+\left(\tilde{I} \otimes D_{23}\right) v_{z}, \\
& H=\left(\tilde{I} \otimes D_{31}\right) v_{x}+\left(\tilde{I} \otimes D_{32}\right) v_{y}+\left(\tilde{I} \otimes D_{33}\right) v_{z},
\end{aligned}
$$


where the notation $\tilde{I}=\left(I_{x} \otimes I_{y} \otimes I_{z}\right)$ and

$$
\begin{aligned}
& v_{x}=\left(D_{x} \otimes I_{y} \otimes I_{z} \otimes I_{M}\right) v \\
& v_{y}=\left(I_{x} \otimes D_{y} \otimes I_{z} \otimes I_{M}\right) v \\
& v_{z}=\left(I_{x} \otimes I_{y} \otimes D_{z} \otimes I_{M}\right) v
\end{aligned}
$$

have been used. The boundary data $g$ is the original boundary data vector of the problem (1) injected on all the gridpoints at the plane $x=1$.

The discrete boundary operators $\tilde{L}^{+}$and $\tilde{L}^{-}$are decomposed as

$$
\begin{aligned}
\tilde{L}^{+} & =\left(I_{x} \otimes I_{y} \otimes I_{z} \otimes L_{0}^{+}\right)+\left(D_{x} \otimes I_{y} \otimes I_{z} \otimes L_{D_{x}}^{+}\right) \\
& +\left(I_{x} \otimes D_{y} \otimes I_{z} \otimes L_{D_{y}}^{+}\right)+\left(I_{x} \otimes I_{y} \otimes D_{z} \otimes L_{D_{z}}^{+}\right) \\
\tilde{L}^{-} & =\left(I_{x} \otimes I_{y} \otimes I_{z} \otimes L_{0}^{-}\right)+\left(D_{x} \otimes I_{y} \otimes I_{z} \otimes L_{D_{x}}^{-}\right) \\
& +\left(I_{x} \otimes D_{y} \otimes I_{z} \otimes L_{D_{y}}^{-}\right)+\left(I_{x} \otimes I_{y} \otimes D_{z} \otimes L_{D_{z}}^{-}\right) .
\end{aligned}
$$

In (31), the matrices $L_{0}^{+}, L_{0}^{-}, L_{D_{x}}^{+}, L_{D_{x}}^{-}, L_{D_{y}}^{+}, L_{D_{y}}^{-}, L_{D_{z}}^{+}$, and $L_{D_{z}}^{-}$correspond to the continuous ones defined in (A.18). In a similar fashion, the matrix $\tilde{R}$ is defined as

$$
\tilde{R}=(\tilde{I} \otimes R) .
$$

where $R$ is the matrix corresponding to the continuous boundary conditions (22). The penalty matrix $\tilde{\Sigma}$ will be chosen such that stability is achieved.

\subsection{Stability}

Regarding stability we rely on the following propositions

Proposition 4. Let the condition (23) hold. If the boundary operators (31) are combined with the penalty matrix

$$
\begin{aligned}
\tilde{\Sigma} & =\left(\tilde{L}^{-}\right)^{T}\left(\tilde{I} \otimes \Lambda_{\mathbf{M}}^{-}\right) \\
& =\left(\left(\tilde{I} \otimes L_{0}^{-}\right)+\left(D_{x} \otimes I_{y} \otimes I_{z} \otimes L_{D_{x}}^{-}\right)\right. \\
& \left.+\left(I_{x} \otimes D_{y} \otimes I_{z} \otimes L_{D_{y}}^{-}\right)+\left(I_{x} \otimes I_{y} \otimes D_{z} \otimes L_{D_{z}}^{-}\right)\right)^{T}\left(\tilde{I} \otimes \Lambda_{\mathbf{M}}^{-}\right)
\end{aligned}
$$

then, the numerical approximation (28) is stable.

Proof. See Appendix B.

Proposition 5. The numerical approximation (28) with boundary operators (31) and penalty coefficients (33) is strongly stable if condition (27) holds.

Proof. See Appendix B. 


\section{A detailed analysis of a model problem}

Consider the simplest possible version of the general problem (1), where

$$
\begin{aligned}
& u=\left[\begin{array}{l}
u_{1} \\
u_{2}
\end{array}\right], \quad A=\left[\begin{array}{cc}
\alpha & 1 \\
1 & \alpha
\end{array}\right], \quad D_{11}=\left[\begin{array}{ll}
0 & 0 \\
0 & \epsilon
\end{array}\right], \\
& \Lambda=\left[\begin{array}{cc}
\alpha+1 & 0 \\
0 & \alpha-1
\end{array}\right], \quad X=\frac{1}{\sqrt{2}}\left[\begin{array}{cc}
1 & -1 \\
1 & 1
\end{array}\right], \quad \epsilon>0
\end{aligned}
$$

where $\alpha \neq \pm 1$ is a real number, $\Lambda$ and $X$ are matrices containing eigenvalues and eigenvectors of $A$, respectively. For simplicity, we consider one stochastic variable, $\vec{\xi}=\xi$. For (34), the continuous boundary conditions defined in (6) are

$$
\begin{aligned}
& W_{0}^{+}-R_{0} W_{0}^{-}=g_{0}^{+}, \\
& W_{1}^{-}-R_{1} W_{1}^{+}=g_{1}^{-},
\end{aligned}
$$

where,

$$
\begin{aligned}
W_{0}^{+} & =\left[\begin{array}{cc}
+1 & 1 \\
0 & 0
\end{array}\right] u_{x=0}-\epsilon\left[\begin{array}{cc}
0 & \frac{1}{(1+\alpha)} \\
\sqrt{2} \alpha & 0
\end{array}\right]\left(u_{x}\right)_{x=0}, \\
W_{0}^{-} & =\left[\begin{array}{ll}
-1 & 1
\end{array}\right] u_{x=0}+\epsilon\left[\begin{array}{ll}
0 & \frac{1}{1-\alpha}
\end{array}\right]\left(u_{x}\right)_{x=0}, \\
W_{1}^{-} & =\left[\begin{array}{ll}
-1 & 1
\end{array}\right] u_{x=1}+\epsilon\left[\begin{array}{cc}
0 & \frac{1}{1-\alpha}
\end{array}\right]\left(u_{x}\right)_{x=1}, \\
W_{1}^{+} & =\left[\begin{array}{cc}
+1 & 1 \\
0 & 0
\end{array}\right] u_{x=1}-\epsilon\left[\begin{array}{cc}
0 & \frac{1}{(1+\alpha)} \\
\sqrt{2} \alpha & 0
\end{array}\right]\left(u_{x}\right)_{x=1} .
\end{aligned}
$$

In (35), $R_{0}$ and $R_{1}$ are vectors of the size $2 \times 1$ and $1 \times 2$, respectively.

\subsection{Accuracy of the model problem}

We start with a quality control. The order of accuracy $p$ is given by

$$
p=\log _{2}\left(\frac{\left\|e_{h}\right\|_{P}}{\left\|e_{\frac{h}{2}}\right\|_{P}}\right), \quad\left\|e_{h}\right\|_{P}^{2}=\left\|u_{a}-u_{h}\right\|_{P}^{2}=\left(u_{a}-u_{h}\right)^{T} P\left(u_{a}-u_{h}\right),
$$

where $e_{h}$ is the error, $u_{h}$ is the numerically computed solution using grid spacing $h$ and data from the manufactured analytical solution $u_{a}$. The analytical solution used producing Table 1 is given by,

$$
u_{a}=\left[\begin{array}{l}
u_{a_{1}} \\
u_{a_{2}}
\end{array}\right]=\left[\begin{array}{l}
\sin (2 \pi(x-t)) \\
\sin (2 \pi(x-t))
\end{array}\right] .
$$

The computed convergence rate $p$ for different SBP-operators and number of grid points in space are shown in Table 1. The rates coincide with the design order of accuracy. 


\begin{tabular}{|c|c|c|c|c|c|}
\hline SBP operator & $N_{x}=20$ & $N_{x}=40$ & $N_{x}=80$ & $N_{x}=160$ & $N_{x}=320$ \\
\hline 2nd order & 1.984 & 1.949 & 1.926 & 1.923 & 1.939 \\
3rd order & 2.942 & 2.862 & 2.841 & 2.867 & 2.909 \\
4th order & 4.561 & 4.364 & 4.017 & 3.893 & 3.904 \\
5th order & 3.885 & 4.238 & 4.295 & 4.596 & 4.919 \\
\hline
\end{tabular}

Table 1: The order of accuracy for the 2nd, 3rd, 4th and 5th order SBP-SAT schemes for different number of grid points in space.

\subsection{Zero variance on the boundary}

In this section we investigate the case with perfect knowledge of the boundary data, i.e. when the variance in the boundary data is zero. The initial and boundary data we prescribe are

$$
\begin{aligned}
g_{0}(t, \xi) & =\left[\begin{array}{l}
3 \\
0
\end{array}\right]-R_{0}, \\
g_{1}(t, \xi) & =1-R_{1}\left[\begin{array}{l}
3 \\
0
\end{array}\right], \\
f(x, \xi) & =\left[3+\xi^{3}, 1-2 \xi\right]^{T} .
\end{aligned}
$$

All values of $R_{0}$ and $R_{1}$ in this section are such that the problem is well-posed and stable. In Figure 1 and 2 , the $L_{1}$-norm of the variance of the solution is illustrated for different choices of boundary conditions and for different distribution of the stochastic variable. As can be seen, the characteristic case with $R_{0}=R_{1}=0$ is clearly the best choice in terms of variance decay, which is in line with the theoretical predictions made in the previous section.

We summarize the results in Table 2 , where the $L_{1}$-norm of the variance is integrated over the whole time domain. Finally, in Table 3 the mean integrated in time and space for uniformly and normally distributed randomness, and different boundary conditions is shown. The same mean is obtained for all cases. We conclude by looking at Table 3 that the type of boundary condition used has no impact on the computed mean of the solution, as stated in Theorem 1. The results from Table 3 is typical for all cases and hence the corresponding results will not be shown for the remaining cases. 


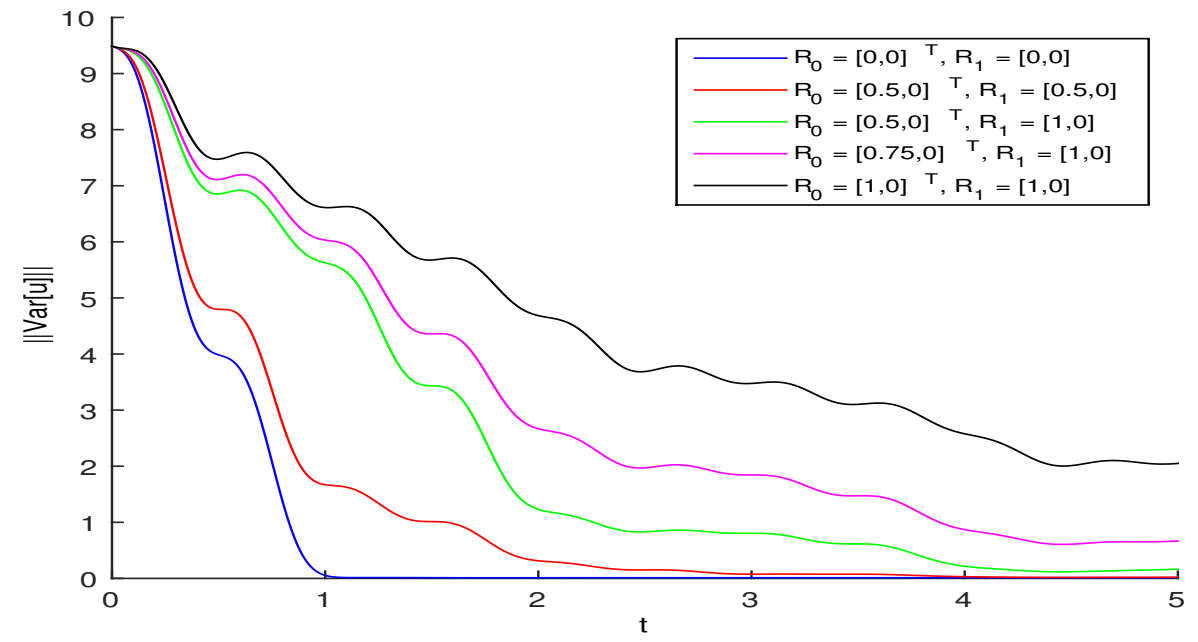

Figure 1: The $L_{1}$-norm of the variance as a function of time for a normally distributed $\xi$ for characteristic and non-characteristic boundary conditions when having perfect boundary knowledge.

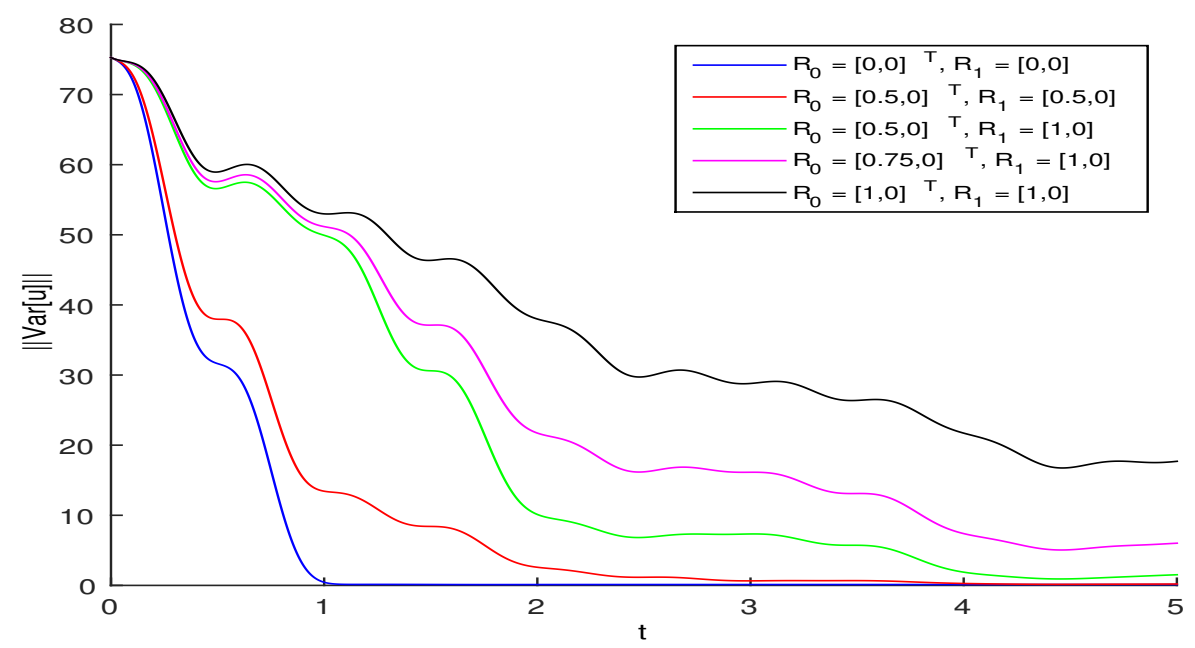

Figure 2: The $L_{1}$-norm of the variance as a function of time for a uniformly distributed $\xi$ for characteristic and non-characteristic boundary conditions when having perfect boundary knowledge. 


\begin{tabular}{|l|l|c|c|c|c|c|}
\hline & $R_{0}, R_{1}=$ & 0,0 & $0.5,0.5$ & $0.5,1$ & $0.75,1$ & 1,1 \\
\hline $\int_{0}^{T}\|\operatorname{Var}[u]\| d t$ & Uniform & 35.320 & 51.900 & 105.570 & 135.008 & 186.022 \\
& Normal & 4.442 & 6.503 & 12.445 & 16.248 & 22.910 \\
\hline
\end{tabular}

Table 2: The integral of the $L_{1}$-norm of the variance for different values of $R_{0}$ and $R_{1}$ for $T=5$.

\begin{tabular}{|c|c|c|c|c|c|c|}
\hline & $R_{0}, R_{1}=$ & 0,0 & $0.5,0.5$ & $0.5,1$ & $0.75,1$ & 1,1 \\
\hline $\int_{0}^{T} \int \mathbb{E}[u] d x d t$ & Uniform & 20.400 & 20.400 & 20.400 & 20.400 & 20.400 \\
& Normal & 20.400 & 20.400 & 20.400 & 20.400 & 20.400 \\
\hline
\end{tabular}

Table 3: The mean integrated in space and time $(T=5)$ for a normally and uniformly distributed $\xi$ for characteristic and non-characteristic boundary conditions, when having perfect boundary knowledge.

\subsection{Decaying variance on the boundary}

For decaying randomness in the boundary data, we choose the following boundary and initial data

$$
\begin{aligned}
g_{0}(t, \xi) & =\left[\begin{array}{c}
3+3 \cos (2 \pi t) e^{-t} \xi^{3}+\frac{\xi}{2(1+\alpha)}\left(2 \cos (2 \pi t) e^{-t} \xi-3 \cos (2 \pi t) e^{-t} \xi^{3}\right) \\
-\frac{\epsilon \alpha}{\sqrt{2}}\left(2 \cos (2 \pi t) e^{-t} \xi-3 \cos (2 \pi t) e^{-t} \xi^{3}\right)
\end{array}\right] \\
& -R_{0}\left[\begin{array}{c}
2+2 \cos (2 \pi t) e^{-t} \xi-\frac{\epsilon}{2(1-\alpha)}\left(2 \cos (2 \pi t) e^{-t} \xi-3 \cos (2 \pi t) e^{-t} \xi^{3}\right)
\end{array}\right], \\
g_{1}(t, \xi) & =\left[\begin{array}{c}
\left.2+2 \cos (2 \pi t) e^{-t} \xi-\frac{\epsilon}{2(1-\alpha)}\left(2 \cos (2 \pi t) e^{-t} \xi-3 \cos (2 \pi t) e^{-t} \xi^{3}\right)\right] \\
-
\end{array}\right], \\
& R_{1}\left[\begin{array}{c}
3+3 \cos (2 \pi t) e^{-t} \xi^{3}+\frac{\xi}{2(1+\alpha)}\left(2 \cos (2 \pi t) e^{-t} \xi-3 \cos (2 \pi t) e^{-t} \xi^{3}\right) \\
-\frac{\epsilon \alpha}{\sqrt{2}}\left(2 \cos (2 \pi t) e^{-t} \xi-3 \cos (2 \pi t) e^{-t} \xi^{3}\right)
\end{array}\right], \\
f(x, \xi) & =\left[3+3 \xi^{3}, 2+2 \xi\right]^{T} .
\end{aligned}
$$

where the added uncertainty in the data has zero mean.

In Figure 3 and 4 the $L_{1}$-norm of the variance of the solution is depicted for different distributions of the stochastic variable, and for different choices of boundary conditions. Figures 3 and 4 clearly show that the characteristic boundary conditions are superior in terms of variance reduction. Finally, we compute the total variance (the $L_{1}$-norm of the variance is integrated over 


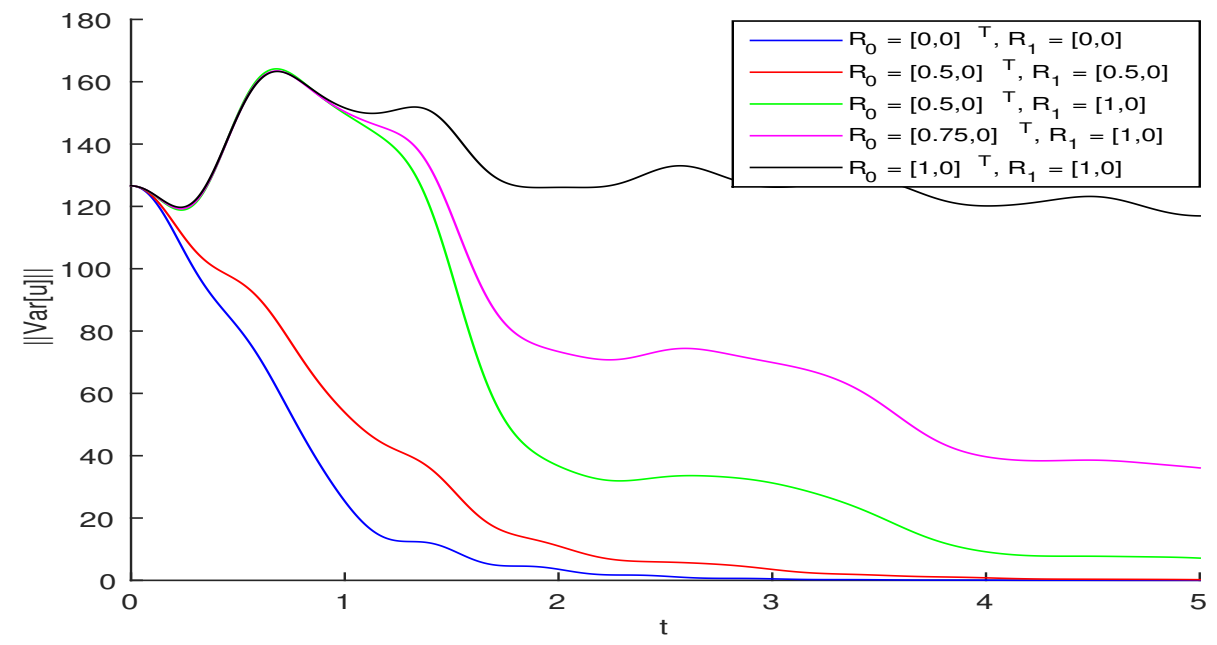

Figure 3: The $L_{1}$-norm of the variance as a function of time for a normally distributed $\xi$ for characteristic and non-characteristic boundary conditions when having decaying boundary data.

\begin{tabular}{|c|c|c|c|c|c|c|}
\hline & $R_{0}, R_{1}=$ & 0,0 & $0.5,0.5$ & $0.5,1$ & $0.75,1$ & 1,1 \\
\hline \multirow{2}{*}{$\int_{0}^{T}\|\operatorname{Var}[u]\| d t$} & Uniform & 135.603 & 196.392 & 418.559 & 561.362 & 800.021 \\
& Normal & 17.940 & 24.840 & 48.991 & 67.982 & 101.266 \\
\hline
\end{tabular}

Table 4: The integral of the $L_{1}$-norm of the variance for different values of $R_{0}$ and $R_{1}$ for $T=5$.

the whole time domain) and illustrate this in Table 4. The computed mean of the solution (not shown) is unaffected by the choice of boundary condition. 


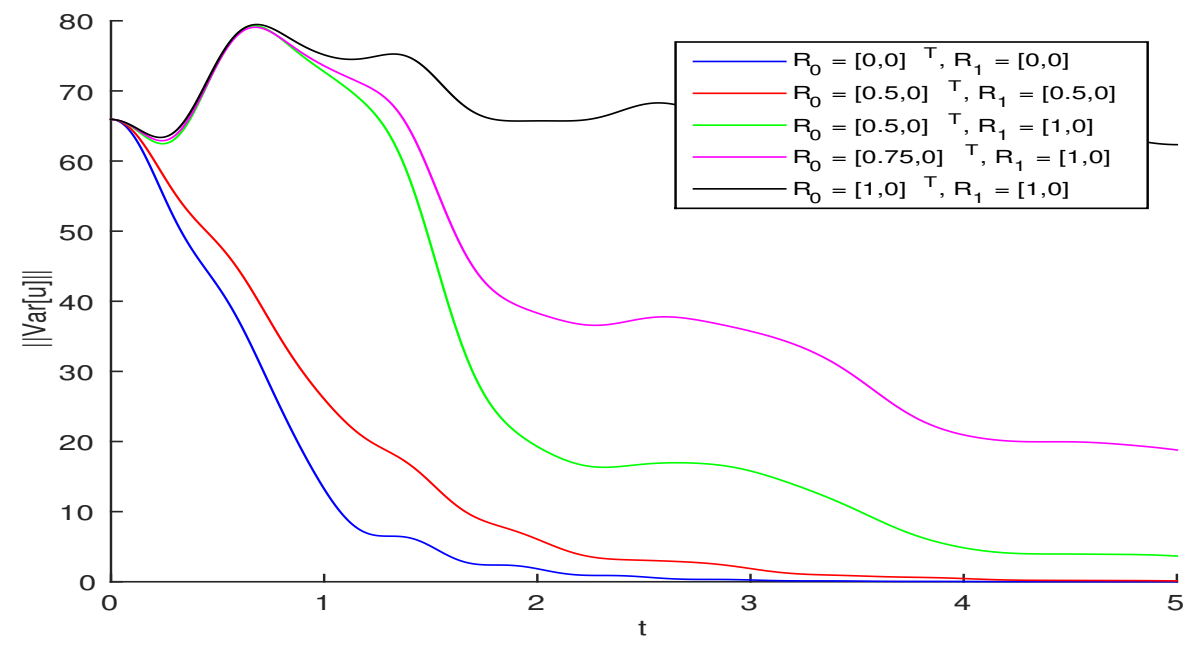

Figure 4: The $L_{1}$-norm of the variance as a function of time for a uniformly distributed $\xi$ for characteristic and non-characteristic boundary conditions when having decaying boundary data.

\subsection{Large non-decaying variance on the boundary}

Finally, for large non-decaying variance on the boundary we choose

$$
\begin{aligned}
& g_{0}(t, \xi)=\left[\begin{array}{c}
3+3 \cos (2 \pi t) \xi^{3}+\frac{\xi}{2(1+\alpha)}\left(2 \cos (2 \pi t) \xi-3 \cos (2 \pi t) \xi^{3}\right) \\
-\frac{\epsilon \alpha}{\sqrt{2}}\left(2 \cos (2 \pi t) \xi-3 \cos (2 \pi t) \xi^{3}\right)
\end{array}\right] \\
& \text { - } R_{0}\left[2+2 \cos (2 \pi t) \xi-\frac{\epsilon}{2(1-\alpha)}\left(2 \cos (2 \pi t) \xi-3 \cos (2 \pi t) \xi^{3}\right)\right], \\
& g_{1}(t, \xi)=\left[2+2 \cos (2 \pi t) \xi-\frac{\epsilon}{2(1-\alpha)}\left(2 \cos (2 \pi t) \xi-3 \cos (2 \pi t) \xi^{3}\right)\right] \\
& -R_{1}\left[\begin{array}{c}
3+3 \cos (2 \pi t) \xi^{3}+\frac{\xi}{2(1+\alpha)}\left(2 \cos (2 \pi t) \xi-3 \cos (2 \pi t) \xi^{3}\right) \\
-\frac{\epsilon \alpha}{\sqrt{2}}\left(2 \cos (2 \pi t) \xi-3 \cos (2 \pi t) \xi^{3}\right)
\end{array}\right], \\
& f(x, \xi)=\left[3+3 \xi^{3}, 2+2 \xi\right]^{T} \text {. }
\end{aligned}
$$

In (40), uncertainty with zero mean is added to the boundary and initial data.

In the figures below we show the $L_{1}$-norm of the variance of the solution for different choices of boundary conditions and different random distributions. Figures 5, 6, 7 and 8 show that the characteristic boundary condition is generally a good choice. We also notice the very large variances for the 


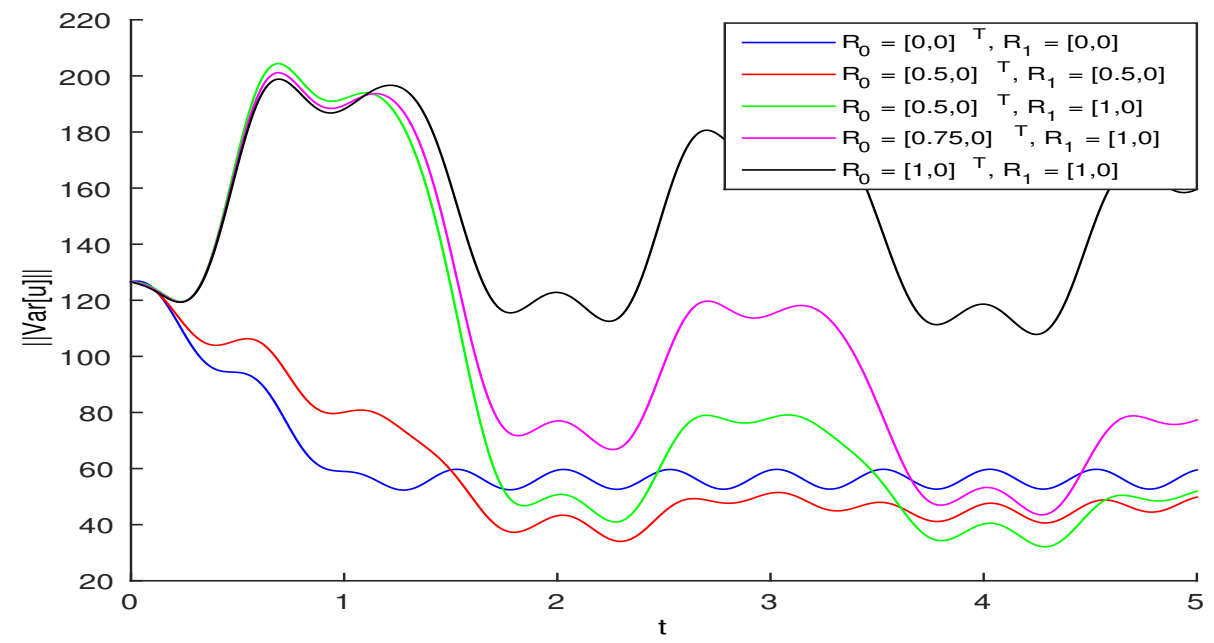

Figure 5: The $L_{1}$-norm of the variance as a function of time for a normally distributed $\xi$ for characteristic and non-characteristic boundary conditions when having large non-decaying boundary data.

\begin{tabular}{|l|l|c|c|c|c|c|}
\hline & $R_{0}, R_{1}=$ & 0,0 & $0.5,0.5$ & $0.5,1$ & $0.75,1$ & 1,1 \\
\hline $\int_{0}^{T}\|\operatorname{Var}[u]\| d t$ & Uniform & 721.313 & 1233.935 & 3318.452 & 4449.668 & 5974.925 \\
& Normal & 91.854 & 133.140 & 343.869 & 464.116 & 629.565 \\
\hline
\end{tabular}

Table 5: The integral of the $L_{1}$-norm of the variance for different values of $R_{0}$ and $R_{1}$ for $T=5$.

cases $R_{0}, R_{1}=1,1, R_{0}, R_{1}=0.75,1$ and $R_{0}, R_{1}=0.5,1$ in practice makes the computations useless.

To quantify the results in figures 5 and 6 , we compute the total variance for all cases and present them in Table 5. The results obtained from computing the mean show, in line with theory, that the choice of boundary condition does not affect the mean value of the solution. In Figure 9, the variance of the solution is shown for different values of the parameter $\epsilon$ using characteristic boundary conditions. Clearly, increasing $\epsilon$, i.e. increasing dissipation, leads to a decrease in the variance. 


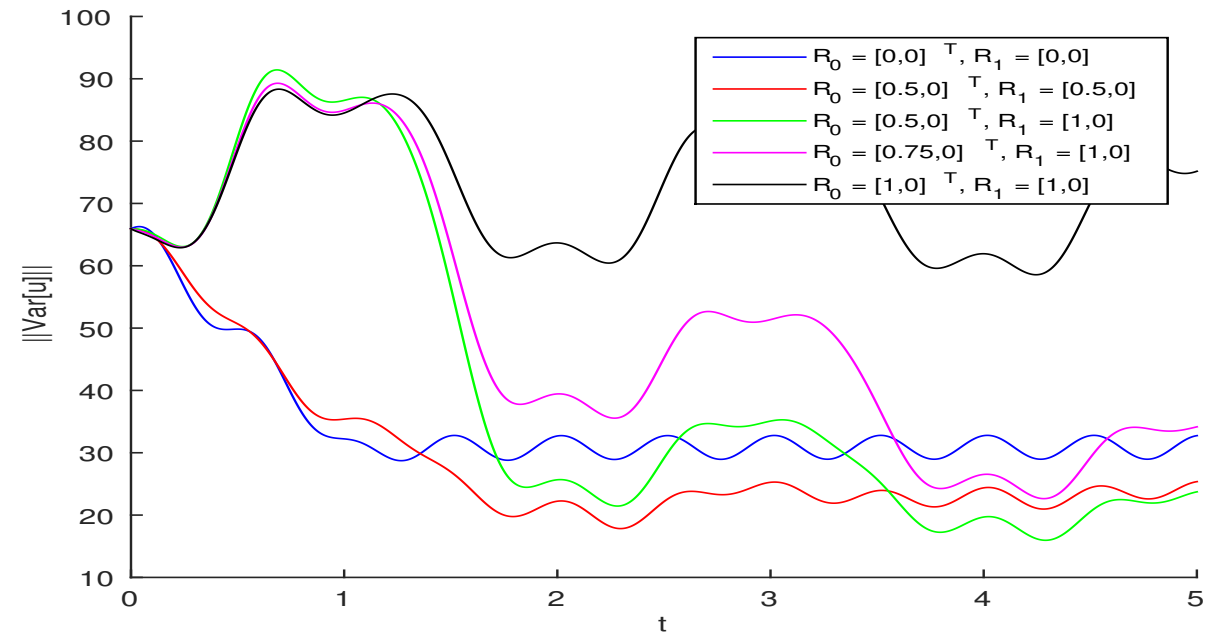

Figure 6: The $L_{1}$-norm of the variance as a function of time for a uniformly distributed $\xi$ for characteristic and non-characteristic boundary conditions when having large nondecaying boundary data.

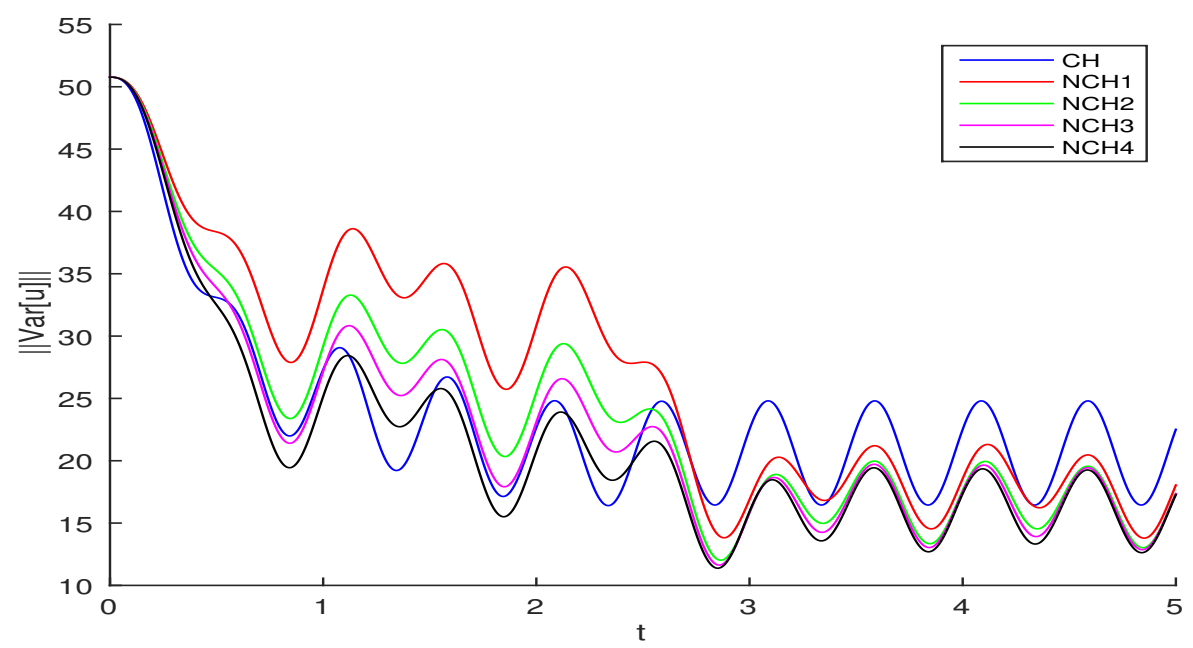

Figure 7: The $L_{1}$-norm of the variance as a function of time for a uniformly distributed $\xi$ for characteristic and non-characteristic boundary conditions (see Appendix D for details) when having large non-decaying boundary data when $\alpha=0.5$. 


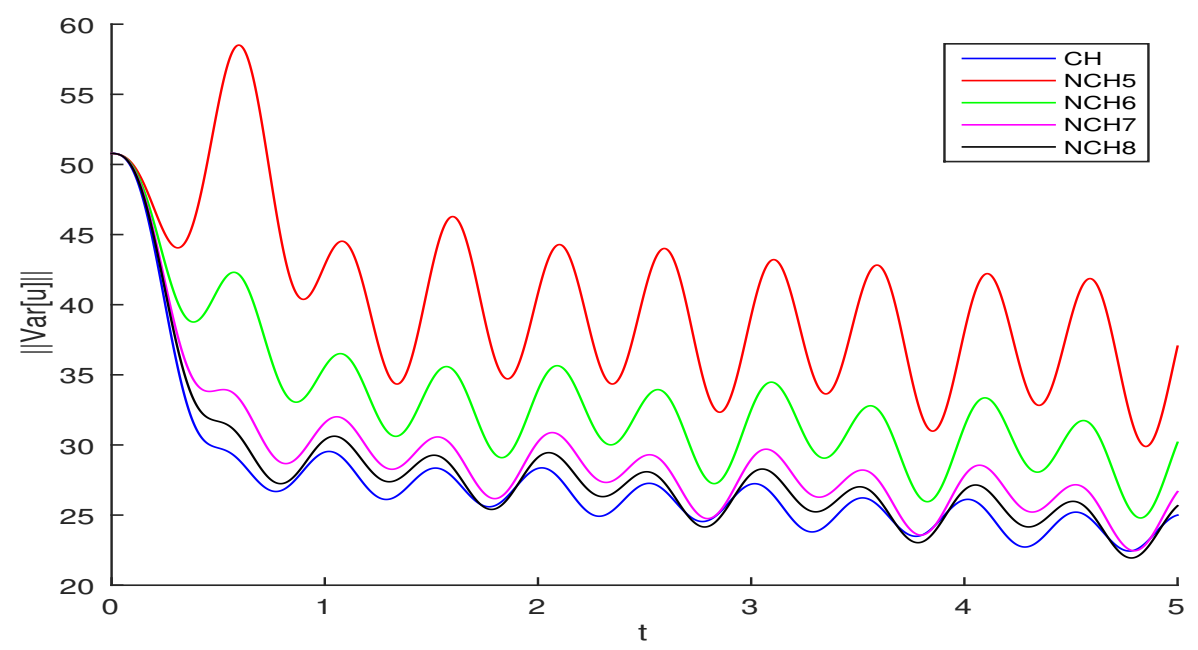

Figure 8: The $L_{1}$-norm of the variance as a function of time for a uniformly distributed $\xi$ for characteristic and non-characteristic boundary conditions (see Appendix D for details) when having large non-decaying boundary data when $\alpha=0.9$.

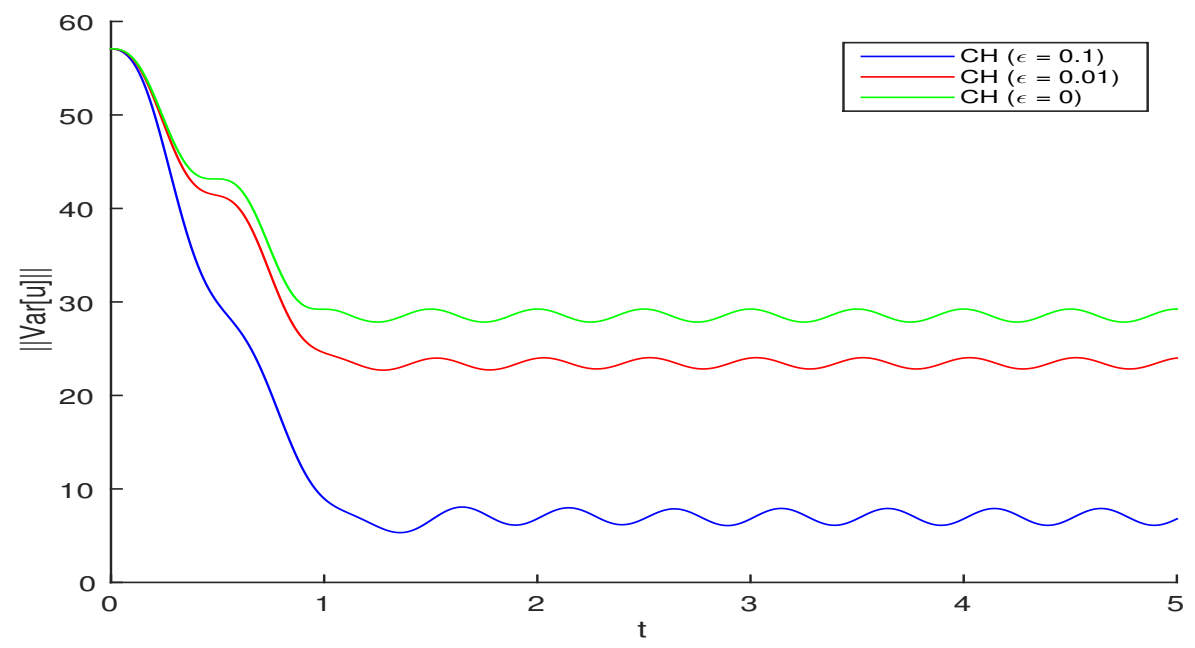

Figure 9: The $L_{1}$-norm of the variance as a function of time for a uniformly distributed $\xi$ for characteristic boundary conditions when having large non-decaying boundary data for the cases $\epsilon=0,0.01$ and 0.1 . 


\section{An application in fluid mechanics}

As a more realistic application we consider the linearized and symmetrized Navier-Stokes equations in one dimension (see [24, 25])

$$
U_{t}+A U_{x}=\epsilon B U_{x x}
$$

where, $U=\left[\frac{\bar{c}}{\sqrt{\gamma} \bar{\rho}} \rho, u, \frac{1}{\bar{c} \sqrt{\gamma(\gamma-1)}} T\right]^{T}$

$$
A=\left[\begin{array}{ccc}
\bar{u} & \frac{\bar{c}}{\sqrt{\gamma}} & 0 \\
\bar{c} & \bar{u} & \sqrt{\frac{\gamma-1}{\gamma}} \bar{c} \\
0 & \sqrt{\frac{\gamma-1}{\gamma}} \bar{c} & \bar{u}
\end{array}\right], \quad B=\left[\begin{array}{ccc}
0 & 0 & 0 \\
0 & \frac{\lambda+2 \mu}{\bar{\rho}} & 0 \\
0 & 0 & \frac{\gamma \mu}{\operatorname{Pr} \bar{\rho}}
\end{array}\right] .
$$

We also use $A=X \Lambda X^{T}, \tilde{B}=X^{T} B X$, where

$$
\tilde{B}=\frac{1}{2}\left[\begin{array}{ccc}
\tilde{\phi} \alpha^{2} & \tilde{\phi} \alpha & \tilde{\phi} \alpha \\
\tilde{\phi} \alpha & \tilde{\phi}+\tilde{\theta} & \tilde{\phi}-\tilde{\theta} \\
\tilde{\phi} \alpha & \tilde{\phi}-\tilde{\theta} & \tilde{\phi}+\tilde{\theta}
\end{array}\right], \quad \tilde{\phi}=\frac{\lambda+2 \mu}{\bar{\rho}}, \quad \tilde{\theta}=\frac{(\gamma-1) \mu}{\operatorname{Pr} \bar{\rho}}, \quad \alpha=\sqrt{\frac{2}{\gamma-1}} .
$$

The variables and parameters are $\rho, u, p, T, c, \gamma, \mu, \lambda, \operatorname{Pr}, \epsilon$ and represent the normalized density, velocity, pressure, temperature, speed of sound, the ratio of specific heat, the dynamic and second viscosity, the Prandtl number and the inverse Reynolds number. The overbar denotes the constant state around which the equations are linearized.

In our calculations we use the following numerical values,

$$
\begin{aligned}
\bar{\rho} & =1, \quad \bar{u}=1, \quad \bar{c}=2, \quad \bar{p}=1, \lambda=-\frac{2}{3}, \\
\gamma & =1.4, \quad \epsilon=0.01, \quad \operatorname{Pr}=0.7, \quad \mu=1 .
\end{aligned}
$$

The parameters (43) models a subsonic flow case. Moerover, the boundary conditions considered are of the type (A.20), where $R_{0}$ and $R_{1}$ are matrices of sizes $3 \times 2$ and $2 \times 3$. The boundary cases which we compare are the 
following

$$
\begin{aligned}
& \text { Characteristic: } R_{0}=\left[\begin{array}{lll}
0 & 0 & 0 \\
0 & 0 & 0
\end{array}\right]^{T}, \quad R_{1}=\left[\begin{array}{lll}
0 & 0 & 0 \\
0 & 0 & 0
\end{array}\right] \text {, } \\
& \text { Specifying } u: R_{0}=\left[\begin{array}{lll}
0 & 0 & 0 \\
0 & 0 & 0
\end{array}\right]^{T}, \quad R_{1}=\left[\begin{array}{ccc}
0 & -1 & r_{13} \\
0 & 0 & r_{23}
\end{array}\right] \text {, } \\
& \text { Specifying } p-\epsilon\left(\frac{\theta}{\bar{\rho}}\right) u_{x}: R_{0}=\left[\begin{array}{lll}
0 & 0 & 0 \\
0 & 0 & 0
\end{array}\right]^{T}, \quad R_{1}=\left[\begin{array}{lll}
0 & 1 & \tilde{r}_{13} \\
0 & 0 & \tilde{r}_{23}
\end{array}\right] \text {, } \\
& \text { Inflow 1: } R_{0}=\left[\begin{array}{ccc}
0 & s_{21} & 0 \\
0 & s_{22} & s_{32}
\end{array}\right]^{T}, \quad R_{1}=\left[\begin{array}{ccc}
0 & 0 & 0 \\
0 & 0 & 0
\end{array}\right] \text {, } \\
& \text { Inflow 2: } R_{0}=\left[\begin{array}{ccc}
\tilde{s}_{11} & 0 & 0 \\
\tilde{s}_{12} & \tilde{s}_{22} & \tilde{s}_{32}
\end{array}\right]^{T}, \quad R_{1}=\left[\begin{array}{ccc}
0 & 0 & 0 \\
0 & 0 & 0
\end{array}\right] \text {. }
\end{aligned}
$$

Note that specifying $u$ and $p-\epsilon(\theta / \bar{\rho}) u_{x}$ are both well-posed boundary conditions at a subsonic outflow boundary, see [26]. The inflow boundary conditions Inflow 1 and Inflow 2 are defined in Appendix C.

The choices of $R_{0}$ and $R_{1}$ in (44) are made such that conditions

$$
R_{0,1}^{T}\left(\Lambda_{\mathbf{M}}\right)_{0,1}^{-} R_{0,1}+\left(\Lambda_{\mathbf{M}}\right)_{0,1}^{+} \geq 0
$$

is satisfied. The randomness imposed in the boundary data is given by

$$
\begin{array}{rlrrr}
\rho & =u=p & = & 1+3 \cos (2 \pi t) \xi_{1}^{3}+3 \sin (2 \pi t) \xi_{2}^{3} \\
\rho_{x} & =u_{x}=p_{x}= & 3 \cos (2 \pi t) \xi_{1}^{3}+3 \sin (2 \pi t) \xi_{2}^{3}
\end{array}
$$

For completeness we give the characteristic variable

$$
w=X^{T} U=\left[\frac{1}{\sqrt{\gamma-1} \bar{\rho} \bar{c}}\left(p-\bar{c}^{2} \rho\right), \frac{1}{\sqrt{2} \bar{\rho} \bar{c}}(p+\bar{c} \bar{\rho} u), \frac{1}{\sqrt{2} \bar{\rho} \bar{c}}(p-\bar{c} \bar{\rho} u)\right]^{T} .
$$

The boundary conditions used in the comparison are two well-known outflow boundary conditions (specifying $u$ and specifying $p-\epsilon\left(\frac{\theta}{\bar{\rho}}\right)$ ) (with characteristic boundary conditions as inflow) and two artificially constructed inflow boundary conditions (see Appendix C for more details) (with characteristic boundary conditions as outflow).

Figures 10 and 11 show the $L_{1}$-norm of the variance for the different cases stated in (44). Tables 6 and 7 illustrate the integrated mean and integrated $L_{1}$-norm of the variance of the solution for the five different boundary conditions. In this case the differences between the various boundary conditions 


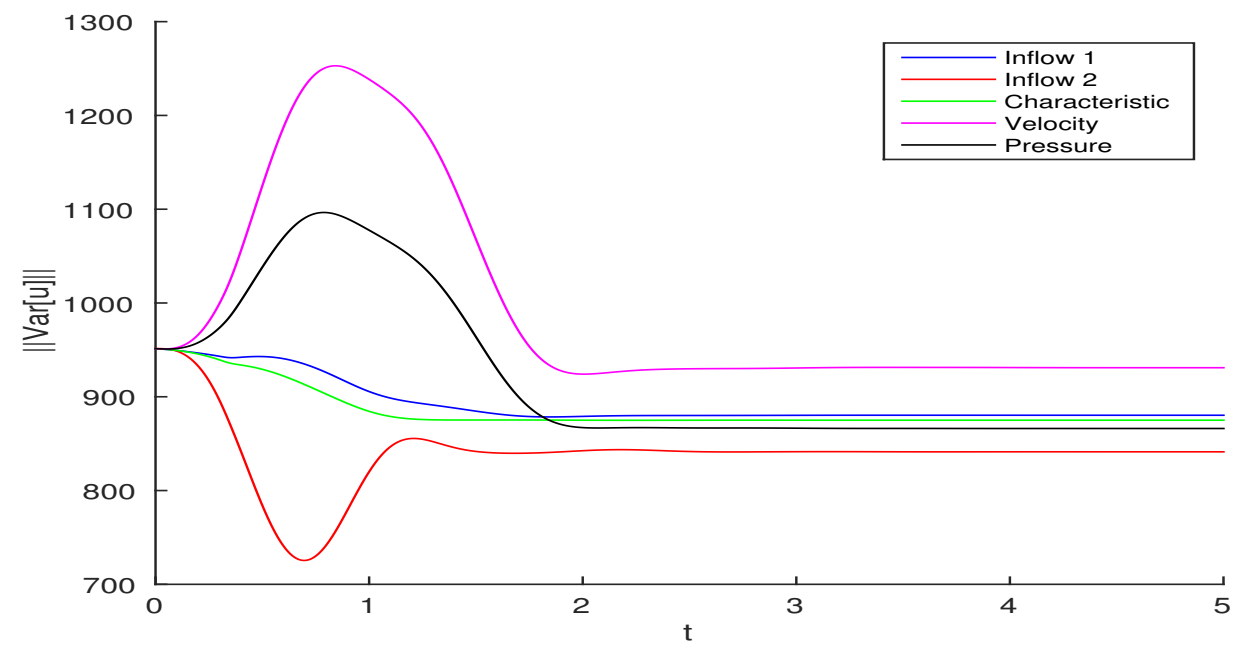

Figure 10: The $L_{1}$-norm of the variance as a function of time for normally distributed $\xi_{1}$ and $\xi_{2}$ for characteristic and non-characteristic boundary conditions when having large non-decaying boundary data for the Navier-Stokes equations.

\begin{tabular}{|c|c|c|c|c|c|c|}
\hline & $B C$ & Charact. & Velocity & Pressure & Inflow 1 & Inflow 2 \\
\hline $\int_{0}^{T} \int \mathbb{E}[u] d x d t$ & Uniform & -8.5311 & -8.5311 & -8.5311 & -8.5311 & -8.5311 \\
& Normal & -8.5311 & -8.5311 & -8.5311 & -8.5311 & -8.5311 \\
\hline
\end{tabular}

Table 6: The mean integrated in space and time $(T=5)$ for different values of $R_{0}$ and $R_{1}$ for $T=5$, and different distributions.

are significantly smaller than for the model problem. From Table 7, we note that the generalized characteristic ( $R=0$ for all boundaries) gives the second lowest total variance. Here, only the boundary condition inflow 2 gives a smaller total variance. From the figures 10 and 11 we observe that the inflow (with characteristics on the outflow) boundary conditions (inflow 1 and inflow 2) provide a significant smaller variance than the outflow (with characteristics on the inflow) boundary conditions (specifying $u$ and specifying $\left.p-\epsilon\left(\frac{\theta}{\bar{\rho}}\right)\right)$. 


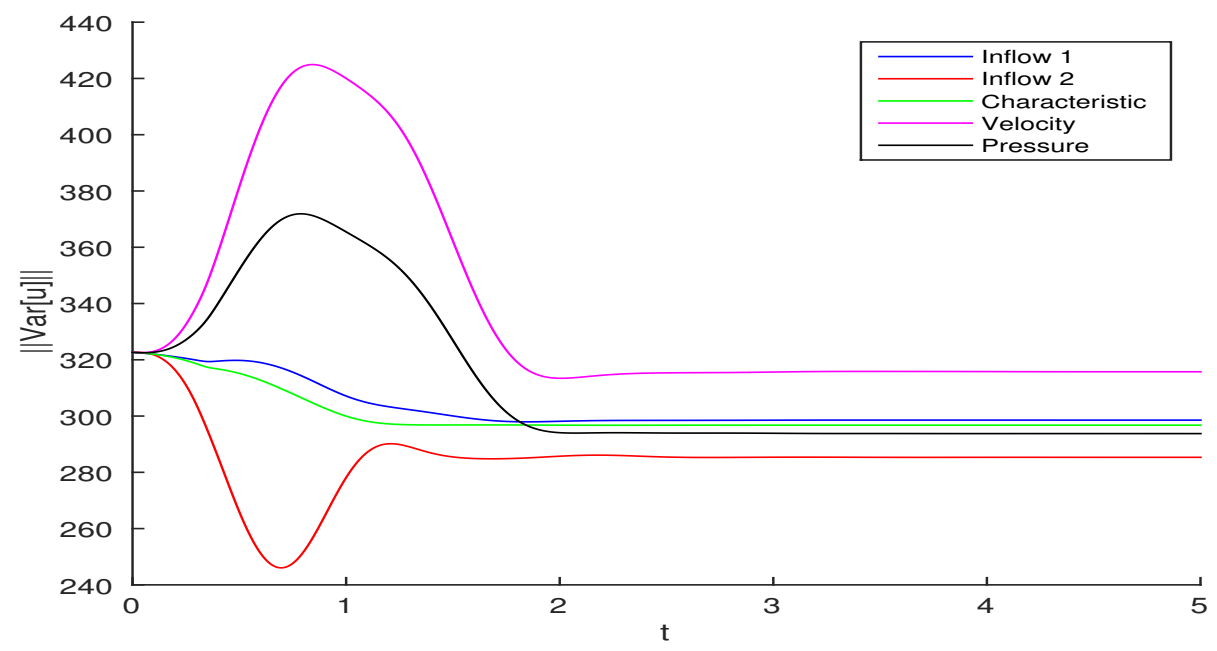

Figure 11: The $L_{1}$-norm of the variance as a function of time for uniformly distributed $\xi_{1}$ and $\xi_{2}$ for characteristic and non-characteristic boundary conditions when having large non-decaying boundary data for the Navier-Stokes equations.

\begin{tabular}{|l|c|c|c|c|c|c|}
\hline & $B C$ & Charact. & Velocity & Pressure & Inflow 1 & Inflow 2 \\
\hline $\int_{0}^{T}\|\operatorname{Var}[u]\| d t$ & Uniform & 1501.8 & 1687.9 & 1558.6 & 1514.8 & 1423.8 \\
& Normal & 4425.8 & 4974.3 & 4593.2 & 4464.2 & 4196.0 \\
\hline
\end{tabular}

Table 7: The integral of the $L_{1}$-norm of the variance for different values of $R_{0}$ and $R_{1}$ for $T=5$, and different distributions. 


\section{Summary and conclusions}

We have studied how the boundary conditions affect the uncertainty of the solution when the initial and boundary data are uncertain. The study has been performed on a general incompletely parabolic system of equations.

A new formulation, an evolution equation, relating the variance of the solution with the boundary condition imposed was derived. It was proven that the expected value of the solution is independent of the choice of boundary condition in the linear case given that the boundary and initial data is extracted from the deterministic solution to the Cauchy problem.

To investigate the relevance of the evolution equation, a model problem of an incompletely parabolic system of equations was studied. Numerical results for three different cases, perfect boundary data, boundary data with decaying variance and boundary data with large non-decaying data were analyzed. Moreover, it was shown that increasing the dissipation leads to a decreasing variance in the solution. This result is natural since the dissipation gives a strictly negative contribution to the variance, which can be seen clearly in the evolution equation for the variance.

The technique was also applied to the one-dimensional Navier-Stokes equations, where the case with large non-decaying variance on the solution was investigated for five different types of boundary conditions. The results were in line with those found in the model problem showing that the generalized characteristic boundary condition was a good choice, with one notable exception.

With given perfect boundary data, we conclude that the optimal choice of boundary operator is the generalized characteristic boundary conditions. For decaying variance in the boundary data, the optimal choice is determined by the different correlations between the inflow and outflow boundary data.

For large non-decaying variance in the boundary data no general conclusions can be drawn, however our numerical results suggests that a good choice is the generalized characteristic boundary conditions, even for this case.

\section{Acknowledgements}

This work was partly done in the UMRIDA project which is supported by the European Commission under contract No. ACP3-GA-2013-605036. 


\section{Appendix A. The continuous problem}

In this section we derive a family of well-posed boundary conditions.

\section{Appendix A.1. The energy method}

Following [45], we multiply (1) by $u^{T}$ and integrate over the domain. By rearranging and defining $\|u\|^{2}=\int_{\Omega} u^{T} u d \Omega$, we obtain

$$
\|u\|_{t}^{2}+2 D I=B T
$$

where,

$$
\begin{aligned}
D I & =\int_{\Omega}\left[\begin{array}{l}
u_{x} \\
u_{y} \\
u_{z}
\end{array}\right]^{T}\left[\begin{array}{lll}
D_{11} & D_{12} & D_{13} \\
D_{21} & D_{22} & D_{23} \\
D_{31} & D_{32} & D_{33}
\end{array}\right]\left[\begin{array}{l}
u_{x} \\
u_{y} \\
u_{z}
\end{array}\right] d \Omega, \\
B T & =-\oint_{\delta \Omega} u^{T} \bar{A} u-2 u^{T} \bar{D} \nabla u d s .
\end{aligned}
$$

In (A.2), $d s=\sqrt{d x^{2}+d y^{2}+d z^{2}}, \nabla u=\left[u_{x}, u_{y}, u_{z}\right]^{T}, \bar{D}=\left[D_{1}, D_{2}, D_{3}\right]$, (note that $\left.\bar{D} \nabla u=D_{1} u_{x}+D_{2} u_{y}+D_{3} u_{z}\right)$ and

$$
\begin{aligned}
& D_{1}=n_{1} D_{11}+n_{2} D_{21}+n_{3} D_{31}, \quad D_{2}=n_{1} D_{12}+n_{2} D_{22}+n_{3} D_{32}, \\
& D_{3}=n_{1} D_{13}+n_{2} D_{23}+n_{3} D_{33}, \quad \bar{A}=n_{1} A+n_{2} B+n_{3} C
\end{aligned}
$$

where $n=\left(n_{1}, n_{2}, n_{3}\right)^{T}$ is the outward pointing unit normal to $\partial \Omega$. The matrices $\bar{A}, D_{1}, D_{2}$ and $D_{3}$ are of size $M \times M$.

Due to the symmetry, $\bar{A}$ can be diagonalized as

$$
\bar{A}=X \Lambda X^{T}, \quad X=\left[X_{+}, X_{-}\right], \quad \Lambda=\left[\begin{array}{cc}
\Lambda^{+} & 0 \\
0 & \Lambda^{-}
\end{array}\right],
$$

where $\Lambda$ containing the eigenvalues of $\bar{A}$ is divided into a positive and negative diagonal block denoted $\Lambda^{+}$and $\Lambda^{-}$, with corresponding eigenvectors $X_{+}$, and $X_{-}$stacked as columns. For simplicity and ease of presentation we assume that no eigenvalue is zero (the case in which the matrix $\bar{A}$ is singular does not cause problems and is ignored for ease of the presentation).

Following [45], Our aim is now to bound the right hand side of (A.1) in terms of data by imposing a minimal number of boundary conditions. That will lead to strong well-posedness, which will be used later in order to bound the variance. See $[23,46]$ for a complete description of the various definitions of well-posedness. 
Appendix A.2. The homogeneous case

We begin by studying the case with zero boundary data, and follow the path of analysis described in [21] for the purely hyperbolic case. Consider

$$
\begin{aligned}
B T & =-\oint_{\delta \Omega} u^{T} \bar{A} u-2 u^{T} \bar{D} \nabla u d s=-\oint_{\delta \Omega}\left[\begin{array}{c}
u \\
\nabla u
\end{array}\right]^{T}\left[\begin{array}{cc}
\bar{A} & -\bar{D} \\
-\bar{D}^{T} & 0
\end{array}\right]\left[\begin{array}{c}
u \\
\nabla u
\end{array}\right] d s \\
& =-\oint_{\delta \Omega} V^{T} \mathbf{M} V d s,
\end{aligned}
$$

where

$$
V=\left[\begin{array}{c}
w-\Lambda^{-1} \tilde{D} \nabla w \\
\nabla w
\end{array}\right], \quad \mathbf{M}=\left[\begin{array}{cc}
\Lambda & 0 \\
0 & K
\end{array}\right] .
$$

The new variables $V$ (a vector of size $4 M \times 1$ ) and the block diagonal matrix M (a matrix of size $4 M \times 4 M)$ was obtained by letting

$$
\begin{aligned}
\tilde{D}_{i} & =X^{T} D_{i} X, \quad \tilde{D}=\left[\tilde{D}_{1}, \tilde{D}_{2}, \tilde{D}_{3}\right]=X^{T} \bar{D}\left(I_{3} \otimes X\right), \\
K & =-\tilde{D}^{T} \Lambda^{-1} \tilde{D}, \quad w=X^{T} u, \quad \nabla w=X^{T} \nabla u .
\end{aligned}
$$

The matrices $\tilde{D}$ and $K$ are of size $M \times 3 M$ and $3 M \times 3 M$ respectively. $I_{3}$ is the identity matrix of size 3 .

Diagonalizing the matrix $\mathbf{M}$ in (A.5) gives us

$$
\mathbf{M}=Y \Lambda_{\mathbf{M}} Y^{T}, \quad Y=\left[Y^{+}, Y^{-}, Y^{0}\right], \quad \Lambda_{\mathbf{M}}=\left[\begin{array}{ccc}
\Lambda_{\mathbf{M}}^{+} & 0 & 0 \\
0 & \Lambda_{\mathbf{M}}^{-} & 0 \\
0 & 0 & 0
\end{array}\right]
$$

where $Y^{+}, Y^{-}$and $Y^{0}$ contain the eigenvectors corresponding to positive, negative and zero eigenvalues of the matrix $\mathbf{M}$. The singularity of the matrix $\mathbf{M}$ is a consequence of the singularities in the matrices $D_{i j}$ in (2). The diagonal matrices $\Lambda_{\mathbf{M}}^{+}$and $\Lambda_{\mathbf{M}}^{-}$contain the positive and negative eigenvalues of $\mathbf{M}$. The diagonalization in (A.7) applied to (A.5) gives

$$
\begin{aligned}
B T & =-\oint_{\delta \Omega}\left(Y^{T} V\right)^{T} \Lambda_{M}\left(Y^{T} V\right) d s=-\oint_{\delta \Omega} W^{T} \Lambda_{M} W d s \\
& =-\oint_{\delta \Omega}\left[\begin{array}{l}
W^{+} \\
W^{-} \\
W^{0}
\end{array}\right]^{T}\left[\begin{array}{ccc}
\Lambda_{\mathbf{M}}^{+} & 0 & 0 \\
0 & \Lambda_{\mathbf{M}}^{-} & 0 \\
0 & 0 & 0
\end{array}\right]\left[\begin{array}{l}
W^{+} \\
W^{-} \\
W^{0}
\end{array}\right] d s
\end{aligned}
$$

In (A.8), we have used the splitting

$$
W=Y^{T} V=\left(\left(Y^{+}\right)^{T} V,\left(Y^{-}\right)^{T} V,\left(Y^{0}\right)^{T} V\right)=\left(W^{+}, W^{-}, W^{0}\right) .
$$


The variables and eigenvalues in (A.8) $\left(W^{0}\right.$ is ignored since it gives zero contribution) can be formulated as

$$
\begin{aligned}
& W^{+}=\left[\begin{array}{c}
w^{+}-D^{+} \nabla w \\
Z_{+}^{T} \nabla w
\end{array}\right], \quad \Lambda_{\mathbf{M}}^{+}=\left[\begin{array}{cc}
\Lambda^{+} & 0 \\
0 & \Lambda_{K}^{+}
\end{array}\right], \\
& W^{-}=\left[\begin{array}{c}
w^{-}-D^{-} \nabla w \\
Z_{-}^{T} \nabla w
\end{array}\right], \quad \Lambda_{\mathbf{M}}^{-}=\left[\begin{array}{cc}
\Lambda^{-} & 0 \\
0 & \Lambda_{K}^{-}
\end{array}\right],
\end{aligned}
$$

where $w^{ \pm}=X_{ \pm}^{T} u$, and $Z_{ \pm}=\left[Z_{1}^{ \pm}, Z_{2}^{ \pm}, Z_{3}^{ \pm}\right]^{T}$ are the eigenvectors corresponding to the positive and negative eigenvalues of the matrix $K$. The matrices $\Lambda_{K}^{+}$and $\Lambda_{K}^{-}$contain the positive and negative eigenvalues of $K$. Further, $D^{+}$ and $D^{-}$are defined as,

$$
D^{ \pm}=\left(\Lambda^{ \pm}\right)^{-1} \tilde{D}^{ \pm}, \quad \text { where } \tilde{D}=\left[\begin{array}{c}
\tilde{D}^{+} \\
\tilde{D}^{-}
\end{array}\right] .
$$

In (A.11), $\tilde{D}^{+}$and $\tilde{D}^{-}$are blocks of the matrix $\tilde{D}$ and have the same number of rows as the number of positive and negative eigenvalues of $\bar{A}$, respectively. The number of columns in $\tilde{D}^{+}$and $\tilde{D}^{-}$is $3 M$.

Equation (A.8) can now be written as

$$
B T=-\oint_{\delta \Omega}\left[\left(W^{+}\right)^{T} \Lambda_{\mathbf{M}}^{+}\left(W^{+}\right)+\left(W^{-}\right)^{T} \Lambda_{\mathbf{M}}^{-}\left(W^{-}\right)\right] d s .
$$

The second matrix in (A.12) is negative definite, and hence we need to bound it by imposing boundary conditions. Next, we choose the general form of boundary conditions,

$$
W^{-}-R W^{+}=0,
$$

where $R$ is a matrix of appropriate size. The number of boundary conditions required is the same as the number of diagonal elements of $\Lambda_{\mathbf{M}}^{-}$(the number of negative eigenvalues of $\bar{A}$ plus the number of negative eigenvalues of $K$ ), which is the same as the number of rows in $R$.

Inserting (A.13) in (A.12) gives

$$
B T=-\oint\left(W^{+}\right)^{T}\left(R^{T} \Lambda_{M}^{-} R+\Lambda_{M}^{+}\right)\left(W^{+}\right) d s
$$

From (A.14), we conclude that

$$
R^{T} \Lambda_{\mathbf{M}}^{-} R+\Lambda_{\mathbf{M}}^{+} \geq 0
$$


is required in order to bound the right-hand side of (A.12). This is in line with the results obtained in [21].

We can now obtain the boundary operator $L$ from (A.13) as,

$$
L=L^{-}-R L^{+},
$$

where

$$
\begin{aligned}
& L^{+}=\left(L_{0}^{+}+L_{D_{x}}^{+} \frac{\partial}{\partial x}+L_{D_{y}}^{+} \frac{\partial}{\partial y}+L_{D_{z}}^{+} \frac{\partial}{\partial z}\right) \\
& L^{-}=\left(L_{0}^{-}+L_{D_{x}}^{-} \frac{\partial}{\partial x}+L_{D_{y}}^{-} \frac{\partial}{\partial y}+L_{D_{z}}^{-} \frac{\partial}{\partial z}\right)
\end{aligned}
$$

and

$$
\begin{aligned}
& L_{0}^{+}=\left[\begin{array}{c}
X_{+}^{T} \\
0
\end{array}\right], \quad L_{0}^{-}=\left[\begin{array}{c}
X_{-}^{T} \\
0
\end{array}\right], \\
& L_{D_{x}}^{+}=\left[\begin{array}{c}
-D_{1}^{+} X_{+}^{T} \\
\left(Z_{1}^{+}\right)^{T} X^{T}
\end{array}\right], \quad L_{D_{x}}^{-}=\left[\begin{array}{c}
-D_{1}^{-} X_{-}^{T} \\
\left(Z_{1}^{-}\right)^{T} X^{T}
\end{array}\right] \text {, } \\
& L_{D_{y}}^{+}=\left[\begin{array}{c}
-D_{2}^{+} X_{+}^{T} \\
\left(Z_{2}^{+}\right)^{T} X^{T}
\end{array}\right], \quad L_{D_{y}}^{-}=\left[\begin{array}{c}
-D_{2}^{-} X_{-}^{T} \\
\left(Z_{2}^{-}\right)^{T} X^{T}
\end{array}\right], \\
& L_{D_{z}}^{+}=\left[\begin{array}{c}
-D_{3}^{+} X_{+}^{T} \\
\left(Z_{3}^{+}\right)^{T} X^{T}
\end{array}\right], \quad L_{D_{z}}^{-}=\left[\begin{array}{c}
-D_{3}^{-} X_{-}^{T} \\
\left(Z_{3}^{-}\right)^{T} X^{T}
\end{array}\right] \text {. }
\end{aligned}
$$

Remark 4. The procedure demonstrated in this section provides the correct number of boundary conditions for the Navier-Stokes equations in 3D. The correct number is given by the number of rows in $L$.

We are now ready to prove Proposition 2

Proof. Time-integration of (A.1) with the boundary conditions (A.13) yields,

$$
\|u(T)\|^{2}+2 \int_{0}^{T} D I d t=\|f\|^{2}+\int_{0}^{T} B T d t
$$

where BT, given by (A.14), is negative semi-definite if (A.15) holds. The estimate (A.19) together with the fact that we use the correct number of boundary conditions implies both uniqueness and existence.

Appendix A.3. The non-homogeneous case

Next, we extend the analysis to the case with non-zero boundary data. By supplying the boundary condition (A.13) with data we have

$$
W^{-}-R W^{+}=g
$$


where $W^{+}, W^{-}$are given in (A.10). By using (A.20) in (A.12), we obtain,

$$
B T=-\oint_{\delta \Omega}\left[\begin{array}{c}
W^{+} \\
g
\end{array}\right]^{T}\left[\begin{array}{cc}
R^{T} \Lambda_{\mathbf{M}}^{-} R+\Lambda_{\mathbf{M}}^{+} & \left(\Lambda_{\mathbf{M}}^{-} R\right)^{T} \\
\Lambda_{\mathbf{M}}^{-} R & \Lambda_{\mathbf{M}}^{-}
\end{array}\right]\left[\begin{array}{c}
W^{+} \\
g
\end{array}\right] d s .
$$

We can now add and subtract $g^{T} G g$, where $G$ is a negative definite matrix of appropriate size, to obtain,

$$
\begin{aligned}
B T= & -\oint_{\delta \Omega}\left[\begin{array}{c}
W^{+} \\
g
\end{array}\right]^{T} \underbrace{\left[\begin{array}{cc}
R^{T} \Lambda_{\mathbf{M}}^{-} R+\Lambda_{\mathbf{M}}^{+} & \left(\Lambda_{\mathbf{M}}^{-} R\right)^{T} \\
\Lambda_{\mathbf{M}}^{-} R & -G
\end{array}\right]}_{\tilde{\mathbf{M}}}\left[\begin{array}{c}
W^{+} \\
g
\end{array}\right] d s \\
& -\oint_{\delta \Omega} g^{T}\left(G+\Lambda_{\mathbf{M}}^{-}\right) g d s,
\end{aligned}
$$

where the second term in (A.22) is bounded by data. For (A.22) to be bounded, the matrix $\tilde{\mathbf{M}}$ must be positive semi-definite.

The matrix $\tilde{\mathbf{M}}$ is symmetric and hence diagonalizable. By rotating $\tilde{\mathbf{M}}$, we obtain

$$
\hat{\mathbf{M}}=C_{0}^{T}\left[\begin{array}{cc}
R^{T} \Lambda_{\mathbf{M}}^{-} R+\Lambda_{\mathbf{M}}^{+} & 0 \\
0 & -G-\left(\Lambda_{\mathbf{M}}^{-} R\right)\left(R^{T} \Lambda_{\mathbf{M}}^{-} R+\Lambda_{\mathbf{M}}^{+}\right)^{-1}\left(\Lambda_{\mathbf{M}}^{-} R\right)^{T}
\end{array}\right] C_{0},
$$

where,

$$
C_{0}=\left[\begin{array}{cc}
I & \left(R^{T} \Lambda_{\mathbf{M}}^{-} R+\Lambda_{\mathbf{M}}^{+}\right)^{-1}\left(\Lambda_{\mathbf{M}}^{-} R\right)^{T} \\
0 & I
\end{array}\right],
$$

and $I$ is the identity matrix. Note that $\tilde{\mathbf{M}}$ is positive semi-definite if $\hat{\mathbf{M}}$ is. To obtain (A.23), a stronger condition than (A.15) is required, we need

$$
R^{T} \Lambda_{\mathbf{M}}^{-} R+\Lambda_{\mathbf{M}}^{+}>0
$$

Also, we note that by choosing the matrix $G$ such that the Schur complement of $\hat{\mathbf{M}}$ is positive semi-definite, i.e

$$
G \leq-\left(\Lambda_{\mathbf{M}}^{-} R\right)\left(R^{T} \Lambda_{\mathbf{M}}^{-} R+\Lambda_{\mathbf{M}}^{+}\right)^{-1}\left(\Lambda_{\mathbf{M}}^{-} R\right)^{T}
$$

we get an energy estimate.

Remark 5. Note that we can always make the choice (A.25) if (A.24) holds. The matrix $G$ is completely arbitrary except for the requirement (A.25). 
We can now prove Proposition 3

Proof. Time-integration of (A.1) subject to (A.20) leads to the following estimate,

$$
\|u(T)\|^{2}+2 \int_{0}^{T} D I d t=\|f\|^{2}+\int_{0}^{T} B T_{c}+g^{T}\left(\left|\Lambda_{\mathbf{M}}^{-}\right|+|G|\right) g d t,
$$

where $B T_{c}$ is given by

$$
B T_{c}=-\left[\begin{array}{c}
W^{+} \\
g
\end{array}\right]^{T}\left[\begin{array}{cc}
R^{T} \Lambda_{\mathbf{M}}^{-} R+\Lambda_{\mathbf{M}}^{+} & \left(\Lambda_{\mathbf{M}}^{-} R\right)^{T} \\
\Lambda_{\mathbf{M}}^{-} R & -G
\end{array}\right]\left[\begin{array}{c}
W^{+} \\
g
\end{array}\right] .
$$

The term $B T_{c}$ in (A.27) is negative semi-definite when $R$ and $G$ are chosen such that (A.24) and (A.25) are satisfied, respectively. By providing these choices, a bound on (A.26) in terms of data is obtained. By imposing the correct number of boundary conditions, uniqueness and existence are guaranteed.

\section{Appendix B. Stability}

To prove stability of the numerical scheme (28), we use the discrete energy method [47, 48], and the SBP properties described above.

\section{Appendix B.1. The homogeneous case}

We multiply (28) by $v^{T}\left(P_{x} \otimes P_{y} \otimes P_{z} \otimes I_{M}\right)$ from the left and add the transpose. By defining the discrete norm $\|v\|_{P_{x y z}}^{2}=v^{T} P_{x y z} v$, where $P_{x y z}=$ $\left(P_{x} \otimes P_{y} \otimes P_{z} \otimes I_{M}\right)$, using the symmetry property of $A$ and the SBP property of $Q_{x, y, z}$, we obtain,

$$
\begin{aligned}
\frac{d}{d t}\|v\|_{P_{x y z}}^{2} & +2 \overline{D I}=-v^{T}\left(E_{N_{x}} \otimes P_{y} \otimes P_{z} \otimes A\right) v \\
& +v_{x}^{T}\left(E_{N_{x}} \otimes P_{y} \otimes P_{z} \otimes D_{11}\right) v \\
& +v^{T}\left(E_{N_{x}} \otimes P_{y} \otimes P_{z} \otimes D_{11}\right) v_{x} \\
& +v_{y}^{T}\left(E_{N_{x}} \otimes P_{y} \otimes P_{z} \otimes D_{12}\right) v \\
& +v^{T}\left(E_{N_{x}} \otimes P_{y} \otimes P_{z} \otimes D_{12}\right) v_{y} \\
& +v_{z}^{T}\left(E_{N_{x}} \otimes P_{y} \otimes P_{z} \otimes D_{13}\right) v \\
& +v^{T}\left(E_{N_{x}} \otimes P_{y} \otimes P_{z} \otimes D_{13}\right) v_{z} \\
& +v^{T}\left(E_{N_{x}} \otimes P_{y} \otimes P_{z} \otimes I_{M}\right) \tilde{\Sigma}\left(\tilde{L}^{-}-\tilde{R} \tilde{L}^{+}\right) v \\
& +v^{T}\left(\tilde{L}^{-}-\tilde{R} \tilde{L}^{+}\right)^{T} \tilde{\Sigma}^{T}\left(E_{N_{x}} \otimes P_{y} \otimes P_{z} \otimes I_{M}\right) v
\end{aligned}
$$


where $v_{N_{x}}$ denotes the numerical solution in the plane at $x=1$ and

$$
\begin{aligned}
\overline{D I} & =\left[\begin{array}{l}
v_{x} \\
v_{y} \\
v_{z}
\end{array}\right]^{T}\left(I_{3} \otimes P_{x y z}\right)\left[\begin{array}{lll}
\tilde{I} \otimes D_{11} & \tilde{I} \otimes D_{12} & \tilde{I} \otimes D_{13} \\
\tilde{I} \otimes D_{21} & \tilde{I} \otimes D_{22} & \tilde{I} \otimes D_{23} \\
\tilde{I} \otimes D_{31} & \tilde{I} \otimes D_{32} & \tilde{I} \otimes D_{33}
\end{array}\right]\left[\begin{array}{c}
v_{x} \\
v_{y} \\
v_{z}
\end{array}\right] \\
& \left.=\left[\begin{array}{c}
v_{x} \\
v_{y} \\
v_{z}
\end{array}\right]^{T}\left(I_{3} \otimes P_{x y z}\right)\left[\Psi^{T}\left(\begin{array}{ccc}
D_{11} & D_{12} & D_{13} \\
D_{21} & D_{22} & D_{23} \\
D_{31} & D_{32} & D_{33}
\end{array}\right\} \otimes \tilde{I}\right) \Psi\right]\left[\begin{array}{c}
v_{x} \\
v_{y} \\
v_{z}
\end{array}\right] .
\end{aligned}
$$

The Kronecker product $\otimes$ is even permutation similar, see [45] for details. Hence, in (B.2), the terms involving the transformation matrix $\Psi$ within the brackets are identical. Note that $\overline{D I}$ is positive semi-definite similarly to its continuous counterpart $D I$ in (A.2).

Next, in a similar way as in the continuous setting (A.7), we introduce

$$
\tilde{W}^{+}=\tilde{L}^{+} v, \quad \tilde{W}^{-}=\tilde{L}^{-} v,
$$

where (B.3) is the discrete version of (A.17). By denoting $P_{y z}=I_{N_{x}} \otimes P_{y} \otimes P_{z}$, and using the definitions of $\tilde{W}^{+}$and $\tilde{W}^{-}$(B.3), in (B.1) we obtain

$$
\begin{aligned}
\frac{d}{d t}\|v\|_{P_{x y z}}^{2}+2 \overline{D I} & =-\left[\begin{array}{c}
\tilde{W}^{+} \\
\tilde{W}^{-}
\end{array}\right]_{N_{x}}^{T}\left(P_{y z} \otimes\left[\begin{array}{cc}
\Lambda_{\mathrm{M}}^{+} & 0 \\
0 & \Lambda_{\mathrm{M}}^{-}
\end{array}\right]\right)\left[\begin{array}{l}
\tilde{W}^{+} \\
\tilde{W}^{-}
\end{array}\right]_{N_{x}} \\
& +v^{T}\left(E_{N_{x}} \otimes P_{y} \otimes P_{z} \otimes I_{M}\right) \tilde{\Sigma}\left(\tilde{L} \tilde{L}^{+}-\tilde{R} \tilde{L}^{-}\right) v \\
& +v^{T}\left(\tilde{L}^{+}-\tilde{R} \tilde{L}^{-}\right)^{T} \tilde{\Sigma}^{T}\left(E_{N_{x}} \otimes P_{y} \otimes P_{z} \otimes I_{M}\right) v .
\end{aligned}
$$

Further, we mimic the analysis in [45], and let $\tilde{\Sigma}=\left(\tilde{L}^{-}\right)^{T} \tilde{\Sigma}^{-}$and $\tilde{\Sigma}^{-}=$ $\left(\tilde{I} \otimes \Sigma^{-}\right)$. By using these relations in (B.4) we obtain,

$$
\begin{aligned}
\frac{d}{d t}\|v\|_{P_{x y z}}^{2} & +2 \overline{D I}= \\
& -\left[\begin{array}{l}
\tilde{W}^{+} \\
\tilde{W}^{-}
\end{array}\right]_{N_{x}}^{T}\left(P_{y z} \otimes\left[\begin{array}{cc}
\Lambda_{\mathbf{M}}^{+} & \left(\Sigma^{-} R\right)^{T} \\
\Sigma^{-} R & \Lambda_{\mathbf{M}}^{-}-\Sigma^{-}-\left(\Sigma^{-}\right)^{T}
\end{array}\right]\right)\left[\begin{array}{l}
\tilde{W}^{+} \\
\tilde{W}^{-}
\end{array}\right]_{N_{x}} .
\end{aligned}
$$

Next, we choose $\Sigma^{-}=\Lambda_{\mathrm{M}}^{-}$, which leads to the final penalty matrix

$$
\tilde{\Sigma}=\left(\tilde{L}^{-}\right)^{T}\left(\tilde{I} \otimes \Lambda_{\mathbf{M}}^{-}\right)
$$

and (B.5) becomes

$$
\frac{d}{d t}\|v\|_{P_{x y z}}^{2}+2 \overline{D I}=-\left[\begin{array}{c}
\tilde{W}^{+} \\
\tilde{W}^{-}
\end{array}\right]_{N_{x}}^{T}\left(P_{y z} \otimes \tilde{S}\right)\left[\begin{array}{c}
\tilde{W}^{+} \\
\tilde{W}^{-}
\end{array}\right]_{N_{x}},
$$


where

$$
\tilde{S}=\left[\begin{array}{cc}
\Lambda_{\mathbf{M}}^{+} & \left(\Lambda_{\mathbf{M}}^{-} R\right)^{T} \\
\Lambda_{\mathbf{M}}^{-} R & -\Lambda_{\mathbf{M}}^{-}
\end{array}\right]
$$

The matrix $\tilde{S}$ is proven positive semi-definite in [45] given that (A.15) holds, and we conclude that the left hand side is bounded.

We are now able to prove Proposition 4

Proof. By integrating (B.5) in time using the penalty parameters defined in (33) we obtain the following estimate,

$$
\|u\|_{P_{x y z}}^{2}+2 \overline{D I}=\|f\|_{P_{x y z}}^{2}+\int_{0}^{T} \overline{B T} d t
$$

where,

$$
\overline{B T}=-\left[\begin{array}{l}
\tilde{W}^{+} \\
\tilde{W}^{-}
\end{array}\right]_{N_{x}}^{T}\left(P_{y z} \otimes \tilde{S}\right)\left[\begin{array}{l}
\tilde{W}^{+} \\
\tilde{W}^{-}
\end{array}\right]_{N_{x}} .
$$

In (B.9), $\overline{B T}$ is negative semi-definite by the fact that $\tilde{S}$ is positive semidefinite, due to (A.15).

Remark 6. Note the similarities between the discrete energy estimate (B.9) and the continuous one (A.19).

Appendix B.2. The non-homogeneous case

Using the same technique as for the homogeneous case, but keeping the boundary data, we end up with the following quadratic form,

$$
\frac{d}{d t}\|v\|_{P_{x y z}}^{2}+2 \overline{D I}=-\left[\begin{array}{c}
\tilde{W}^{+} \\
\tilde{W}^{-} \\
g
\end{array}\right]_{N_{x}}^{T}\left(P_{y z} \otimes \tilde{T}\right)\left[\begin{array}{c}
\tilde{W}^{+} \\
\tilde{W}^{-} \\
g
\end{array}\right]_{N_{x}}
$$

where

$$
\tilde{T}=\left[\begin{array}{ccc}
\Lambda_{\mathbf{M}}^{+} & \left(\Lambda_{\mathbf{M}}^{-} R\right)^{T} & 0 \\
\Lambda_{\mathbf{M}}^{-} R & -\Lambda_{\mathbf{M}}^{-} & \Lambda_{\mathbf{M}}^{-} \\
0 & \Lambda_{\mathbf{M}}^{-} & 0
\end{array}\right]
$$

The matrix $\tilde{T}$ can be split into three parts as

$$
\tilde{T}=\tilde{T}_{1}+\left[\begin{array}{ccc}
0 & 0 & 0 \\
0 & 0 & 0 \\
0 & 0 & -G+\Lambda^{-}
\end{array}\right]
$$


where

$$
\tilde{T}_{1}=\left[\begin{array}{ccc}
-R^{T} \Lambda_{\mathbf{M}}^{-} R & R^{T} \Lambda_{\mathbf{M}}^{-} & -R^{T} \Lambda_{\mathbf{M}}^{-} \\
\Lambda_{\mathbf{M}}^{-} R & -\Lambda_{\mathbf{M}}^{-} & \Lambda_{\mathbf{M}}^{-} \\
-\Lambda_{\mathbf{M}}^{-} R & \Lambda_{\mathbf{M}}^{-} & -\Lambda_{\mathbf{M}}^{-}
\end{array}\right]+\left[\begin{array}{ccc}
R^{T} \Lambda_{\mathbf{M}}^{-} R+\Lambda_{\mathbf{M}}^{+} & 0 & R^{T} \Lambda_{\mathbf{M}}^{-} \\
0 & 0 & 0 \\
\Lambda_{\mathbf{M}}^{-} R & 0 & G
\end{array}\right] .
$$

Similarly to the continuous case, we have added and subtracted $G$ which is an arbitrary matrix used in order to prove stability. In [45], it was shown that $\tilde{T}_{1}$ is positive semi-definite provided that $G$ is chosen appropriately, and hence, the left-hand side of (B.10) is bounded and the numerical approximation strongly stable.

We can now prove Proposition 5,

Proof. Integrating (B.10) in time gives us,

$$
\|u\|_{P_{x y z}}^{2}+2 \int_{0}^{T} \overline{D I} d t=\|f\|_{P_{x y z}}^{2}+\int_{0}^{T}\left[B T+g_{N_{x}}^{T}\left(\left|\Lambda_{\mathbf{M}}^{-}\right|+|G|\right) g_{N_{x}}\right] d t,
$$

with

$$
B T=-\left[\begin{array}{c}
\tilde{W}^{+} \\
\tilde{W}^{-} \\
g
\end{array}\right]_{N_{x}}^{T}\left(P_{y z} \otimes \tilde{T}_{1}\right)\left[\begin{array}{c}
\tilde{W}^{+} \\
\tilde{W}^{-} \\
g
\end{array}\right]_{N_{x}} \leq 0,
$$

which concludes the proof.

Remark 7. Note the similarity between the estimate (B.14) and the continuous counterpart (A.26).

\section{Appendix C. Inflow boundary conditions}

The inflow boundary conditions Inflow 1 and Inflow 2 are constructed by choosing $R_{0}$ to achieve well-posedness, and specified as

Inflow 1:

$$
\begin{aligned}
\left(p-\bar{c}^{2} \rho\right) \frac{1}{\bar{c} \sqrt{\gamma-1} \bar{\rho}}-\left(p_{x}-\frac{\bar{c}^{2}}{\gamma} \rho_{x}\right) \frac{\gamma \epsilon \mu}{\bar{c} \operatorname{Pr} \bar{u} \sqrt{\gamma-1} \bar{\rho}} & =g_{1}(t) \\
p\left(\frac{2 \sqrt{\bar{c}+\bar{u}}-\sqrt{\bar{c}-\bar{u}}}{2 \sqrt{2} \bar{c} \bar{\rho} \sqrt{\bar{c}+\bar{u}}}\right)+u\left(\frac{2 \sqrt{\bar{c}+\bar{u}}+\sqrt{\bar{c}-\bar{u}}}{2 \sqrt{2} \bar{c} \bar{\rho} \sqrt{\bar{c}+\bar{u}}}\right) & - \\
\left(p_{x}-\frac{\bar{c}^{2}}{\gamma} \rho_{x}\right)\left(\frac{\gamma \epsilon \mu\left(2 \sqrt{\bar{c}-\bar{u}}+\sqrt{\bar{c}+\bar{u}}+2 \operatorname{Pr}^{2} \bar{u} \bar{c} \sqrt{\bar{c}+\bar{u}}(\lambda+2 \mu)^{2}\right)}{\bar{c} \sqrt{\bar{c}-\bar{u}}\left(C_{1}-C_{6}+C_{2}\right)}\right. & =g_{2}(t) \\
\left(p_{x}-\frac{\bar{c}^{2}}{\gamma} \rho_{x}\right) \frac{C_{7}}{\left(-C_{5}-C_{6}\right)\left(C_{5}+C_{2}\right) \bar{\rho}} & =g_{3}(t) .
\end{aligned}
$$


Inflow 2:

$$
\begin{aligned}
& p\left(\frac{1}{\bar{c} \bar{\rho} \sqrt{\gamma-1}}-\frac{\sqrt{\bar{c}-\bar{u}}}{2 \sqrt{2} \bar{c} \bar{\rho} \sqrt{\bar{u}}}\right)+u \frac{\sqrt{\bar{c}-\bar{u}}}{2 \sqrt{2 \bar{u}}}-\rho \frac{\bar{c}}{\sqrt{\gamma-1} \bar{\rho}}+ \\
& \left(p_{x}-\bar{c}^{2} \rho_{x}\right) \frac{\epsilon \bar{c} \mu}{4 \operatorname{Pr} \bar{\rho}^{2}}\left(\frac{4-\sqrt{2(\bar{c}-\bar{u})(\gamma-1)}}{\bar{u} \sqrt{\gamma-1}}-\frac{C_{4}}{(\bar{c}-\bar{u})\left(C_{5}+C_{2}\right)}\right)=g_{1}(t) \\
& \frac{p}{\sqrt{2} \bar{c} \bar{\rho}}+\frac{u}{\sqrt{2}}+\left(p_{x}-\frac{\bar{c}^{2}}{\gamma} \rho_{x}\right) \frac{\epsilon\left(C_{1}-C_{2}-C_{6}\right)}{2 \sqrt{2} \bar{c}^{2} \operatorname{Pr} \bar{u}(\bar{c}+\bar{u})(\gamma-1) \mu \bar{\rho}^{2}}=g_{2}(t) \\
& \left(p_{x}-\frac{\bar{c}^{2}}{\gamma} \rho_{x}\right) \frac{C_{7}}{\left(-C_{5}-C_{6}\right)\left(C_{5}+C_{2}\right) \bar{\rho}}=g_{3}(t) .
\end{aligned}
$$

In (44), the following notations are used

$$
\begin{aligned}
r_{13} & =\frac{C_{1}-C_{2}}{C_{3} \bar{c}} & r_{23} & =\frac{C_{1}-C_{2}}{C_{1}+C_{2}} \\
\tilde{r}_{13} & =\frac{C_{1}-C_{2}}{C_{3} \bar{u}} & \tilde{r}_{23} & =\frac{C_{1}-C_{2}}{C_{1}+C_{2}} \\
s_{21} & =\frac{1}{2} \sqrt{\frac{\bar{c}-\bar{u}}{\bar{c}+\bar{u}}} & s_{22} & =\left(2-\sqrt{\frac{\bar{c}-\bar{u}}{\bar{c}+\bar{u}}}\right)(\lambda+2 \mu) \frac{C_{1}+C_{2}}{4(\bar{c}+\bar{u})\left(C_{1}-C_{6}+C_{2}\right) \bar{\rho}} \\
\tilde{s}_{11} & =\frac{1}{2} \sqrt{\frac{\bar{c}-\bar{u}}{\bar{u}}} & \tilde{s}_{12} & =-\sqrt{\frac{\bar{c}-\bar{u}}{\bar{u}}}(\lambda+2 \mu) \frac{C_{1}+C_{2}}{4(\bar{c}-\bar{u})\left(C_{1}-C_{6}+C_{2}\right) \bar{\rho}} \\
\tilde{s}_{22} & =\frac{C_{1}+C_{2}}{4 \bar{c} \operatorname{Pr} \bar{u}(\bar{c}+\bar{u}) \gamma \mu \bar{\rho}} & s_{32} & =\tilde{s}_{32}=\frac{\left(C_{5}-C_{2}\right)\left(C_{5}+C_{6}+C_{2}\right)}{\left(C_{2}-C_{5}-C_{6}\right)\left(C_{5}+C_{2}\right)},
\end{aligned}
$$

where,

$$
\begin{aligned}
& C_{1}=\gamma\left(\bar{c}^{2}-\bar{u}^{2} \gamma\right) \mu^{2}+2 \bar{c} \operatorname{Pr} \bar{u}(\gamma-1) \mu(\lambda+2 \mu)+(\operatorname{Pr} \bar{u}(\lambda+2 \mu))^{2} \\
& C_{2}=\sqrt{\left(\gamma\left(\bar{c}^{2}-\bar{u}^{2} \gamma\right) \mu^{2}\right)^{2}-2 \operatorname{Pr}^{2} \bar{u}^{2} \gamma\left(\bar{c}^{2}-2 \bar{c}^{2} \gamma+\bar{u}^{2} \gamma\right) \mu^{2}(\lambda+2 \mu)^{2}+(\operatorname{Pr} \bar{u}(\lambda+2 \mu))^{4}} \\
& C_{3}=2 \operatorname{Pr}^{2}\left(\bar{c}^{2}-\bar{u}^{2}\right)(\lambda+2 \mu) \bar{\rho} \\
& C_{4}=2 \bar{c} \operatorname{Pr} \sqrt{\bar{c}-2 \bar{u}}(\lambda+2 \mu)^{2} \\
& C_{5}=\gamma\left(\bar{c}^{2}-\bar{u}^{2} \gamma\right) \mu^{2}+(\operatorname{Pr} \bar{u}(\lambda+2 \mu))^{2} \\
& C_{6}=2 \bar{c} \operatorname{Pr} \bar{u}(\gamma-1) \mu(\lambda+2 \mu) \\
& C_{7}=4 \sqrt{2} \epsilon \operatorname{Pr} \bar{u} \gamma \mu(\lambda+2 \mu)^{2}\left(\gamma\left(\bar{u}^{2} \gamma-\bar{c}^{2}\right) \mu^{2}-\operatorname{Pr}^{2} \bar{u}^{2}(\lambda+2 \mu)^{2}\right) .
\end{aligned}
$$




\section{Appendix D. Boundary cases}

The boundary cases stated in the figures 7 and 8 are

$$
\begin{aligned}
& \mathrm{CH}: \quad R_{0}=[0,0]^{T}, \quad R_{1}=[0,0] \\
& \mathrm{NCH} 1: \quad R_{0}=\left[\frac{1}{3}, \sqrt{\frac{1}{2}}\right]^{T}, \quad R_{1}=\left[\sqrt{\frac{3}{4}}, 1\right] \\
& \mathrm{NCH} 2: \quad R_{0}=\left[\sqrt{\frac{1}{12}}, \frac{1}{2}\right]^{T}, \quad R_{1}=\left[\sqrt{\frac{1}{2}}, \sqrt{\frac{1}{2}}\right] \\
& \mathrm{NCH} 3: \quad R_{0}=\left[\sqrt{\frac{1}{15}}, \sqrt{\frac{1}{6}}\right]_{T}^{T}, \quad R_{1}=\left[\sqrt{\frac{3}{8}}, \frac{1}{2}\right] \\
& \text { NCH4: } \quad R_{0}=\left[\sqrt{\frac{1}{18}}, \sqrt{\frac{1}{8}}\right]_{T}^{T}, \quad R_{1}=\left[\frac{1}{2}, \sqrt{\frac{1}{6}}\right] \\
& \mathrm{NCH5}: \quad R_{0}=\left[\sqrt{\frac{10}{361}}, \frac{1}{10}\right]^{T}, \quad R_{1}=\left[\sqrt{10}, \sqrt{\frac{8100}{361}}\right] \\
& \text { NCH6: } \quad R_{0}=\left[\sqrt{\frac{5}{361}}, \sqrt{\frac{1}{200}}\right]^{T}, \quad R_{1}=\left[\sqrt{5}, \sqrt{\frac{4050}{361}}\right] \\
& \mathrm{NCH7}: \quad R_{0}=\left[\sqrt{\frac{2}{361}}, \frac{1}{20}\right]^{T}, \quad R_{1}=\left[\sqrt{2}, \sqrt{\frac{2025}{361}}\right] \\
& \text { NCH8: } \quad R_{0}=\left[\sqrt{\frac{1}{361}}, \sqrt{\frac{1}{600}}\right]^{T}, \quad R_{1}=\left[1, \sqrt{\frac{1000}{361}}\right]
\end{aligned}
$$

\section{References}

[1] S. Poroseva, J. Letschert, M. Y. Hussaini, Uncertainty quantification in hurricane path forecasts using evidence theory, in: APS Division of Fluid Dynamics Meeting Abstracts.

[2] M. T. Reagan, H. N. Najm, R. G. Ghanem, O. M. Knio, Uncertainty quantification in reacting-flow simulations through non-intrusive spectral projection, COMBUST FLAME (2003) 545-555.

[3] R. Ghanem, S. Dham, Stochastic finite element analysis for multiphase flow in heterogeneous porous media, TRANSPORT POROUS MED 32 (1998) 239-262.

[4] M. Christie, V. Demyanov, D. Erbas, Uncertainty quantification for porous media flows, J COMPUT PHYS 217 (2006) 143-158.

[5] Z. Zeng, J.-M. Jin, Efficient calculation of scattering variation due to uncertain geometrical deviation, ELECTROMAGNETICS 27 (2007) 387-398. 
[6] C. Chauvière, J. S. Hesthaven, L. Lurati, Computational modeling of uncertainty in time-domain electromagnetics, SIAM J SCI COMPUT 28 (2006) 751-775.

[7] C. M. Sayers, L. D. den Boer, Z. R. Nagy, P. J. Hooyman, Wellconstrained seismic estimation of pore pressure with uncertainty, The Leading Edge 25 (2006) 1524-1526.

[8] P. Thore, A. Shtuka, M. Lecour, T. Ait-Ettajer, R. Cognot, Structural uncertainties: Determination, management, and applications, GEOPHYSICS 67 (2002) 840-852.

[9] H. N. Najm, M. Valorani, Enforcing positivity in intrusive PC-UQ methods for reactive ode systems, J COMPUT PHYS 270 (2014) 544-569.

[10] G. Lin, A. M. Tartakovsky, D. M. Tartakovsky, Uncertainty quantification via random domain decomposition and probabilistic collocation on sparse grids, J COMPUT PHYS 229 (2010) 6995-7012.

[11] T. Barth, On the propagation of statistical model parameter uncertainty in CFD calculations, THEOR COMP FLUID DYN 26 (2012) 435-457.

[12] J. Tryoen, O. Le Maitre, M. Ndjinga, A. Ern, Intrusive Galerkin methods with upwinding for uncertain nonlinear hyperbolic systems, J COMPUT PHYS 229 (2010) 6485-6511.

[13] S. Corveleyn, E. Rosseel, S. Vandewalle, Iterative solvers for a spectral Galerkin approach to elliptic partial differential equations with fuzzy coefficients, SIAM J SCI COMPUT 35 (2013) S420-S444.

[14] P. Pettersson, J. Nordström, A. Doostan, A well-posed and stable stochastic Galerkin formulation of the incompressible Navier-Stokes equations with random data, J COMPUT PHYS 306 (2016) 92-116.

[15] S. Acharjee, N. Zabaras, A non-intrusive stochastic Galerkin approach for modeling uncertainty propagation in deformation processes, COMPUT STRUCT 85 (2007) 244-254.

[16] O. Knio, O. Le Maitre, Uncertainty propagation in CFD using polynomial chaos decomposition, FLUID DYN RES 38 (2006) 616-640. 
[17] W. J. Morokoff, R. E. Caflisch, Quasi-monte carlo integration, J COMPUT PHYS 122 (1995) 218-230.

[18] M. Grigoriu, Stochastic calculus: applications in science and engineering, Springer Science \& Business Media, 2013.

[19] M. Griebel, Adaptive sparse grid multilevel methods for elliptic PDEs based on finite differences, COMPUTING 61 (1998) 151-179.

[20] T. Gerstner, M. Griebel, Numerical integration using sparse grids, Numerical algorithms 18 (1998) 209-232.

[21] J. Nordström, M. Wahlsten, Variance reduction through robust design of boundary conditions for stochastic hyperbolic systems of equations, J COMPUT PHYS 282 (2015) 1-22.

[22] J. C. Strikwerda, Initial boundary value problems for incompletely parabolic systems, COMMUN PUR APPL MATH 30 (1977) 797-822.

[23] J. Nordström, M. Svärd, Well-posed boundary conditions for the Navier-Stokes Equations, SIAM J NUMER ANAL 43 (2005) 12311255 .

[24] J. Berg, J. Nordström, Duality based boundary conditions and dual consistent finite difference discretizations of the Navier-Stokes and Euler equations, J COMPUT PHYS 259 (2014) 135-153.

[25] J. Nordström, The use of characteristic boundary conditions for the Navier-Stokes equations, COMPUT FLUIDS 24 (1995) 609-623.

[26] J. Nordström, The influence of open boundary conditions on the convergence to steady state for the Navier-Stokes equations, J COMPUT PHYS 85 (1989) 210-244.

[27] B. Strand, Summation by parts for finite difference approximations for d/dx, J COMPUT PHYS 110 (1994) 47-67.

[28] H.-O. Kreiss, G. Scherer, Finite element and finite difference methods for hyperbolic partial differential equations, Mathematical aspects of finite elements in partial differential equations (1974) 195-212. 
[29] M. Carpenter, J. Nordström, D. Gottlieb, A Stable and Conservative Interface Treatment of Arbitrary Spatial Accuracy, J COMPUT PHYS 148 (1999) 341-365.

[30] K. Mattsson, J. Nordström, Summation by parts operators for finite difference approximations of second derivatives, J COMPUT PHYS 199 (2004) 503-540.

[31] M. H. Carpenter, D. Gottlieb, S. Abarbanel, Time-stable boundary conditions for finite-difference schemes solving hyperbolic systems: methodology and application to high-order compact schemes, J COMPUT PHYS 111 (1994) 220-236.

[32] M. Svärd, M. H. Carpenter, J. Nordström, A stable high-order finite difference scheme for the compressible Navier-Stokes equations, far-field boundary conditions, J COMPUT PHYS 225 (2007) 1020-1038.

[33] J. Berg, J. Nordström, Stable robin solid wall boundary conditions for the Navier-Stokes equations, J COMPUT PHYS 230 (2011) 7519-7532.

[34] V. Barthelmann, E. Novak, K. Ritter, High dimensional polynomial interpolation on sparse grids, ADV COMPUT MATH 12 (2000) 273288.

[35] U. Banerjee, M. Suri, The effect of numerical quadrature in the $p$-version of the finite element method, MATH COMPUT 59 (1992) 1-20.

[36] L. Gilli, D. Lathouwers, J. Kloosterman, T. van der Hagen, A. Koning, D. Rochman, Uncertainty quantification for criticality problems using non-intrusive and adaptive polynomial chaos techniques, ANN NUCL ENERGY 56 (2013) 71-80.

[37] J. He, S. Gao, J. Gong, A sparse grid stochastic collocation method for structural reliability analysis, STRUCT SAF 51 (2014) 29-34.

[38] J. Nordström, B. Lönn, Energy decay of vortices in viscous fluids: an applied mathematics view, J FLUID MECH 709 (2012) 593-609.

[39] P. Pettersson, G. Iaccarino, J. Nordström, A stochastic Galerkin method for the Euler equations with Roe variable transformation, J COMPUT PHYS 257 (2014) 481-500. 
[40] B. Gustafsson, High order difference methods for time dependent PDE, volume 38, Springer Science \& Business Media, 2007.

[41] K. Mattsson, M. Almquist, M. H. Carpenter, Optimal diagonal-norm SBP operators, J COMPUT PHYS 264 (2014) 91-111.

[42] J. E. Hicken, D. W. Zingg, Summation-by-parts operators and highorder quadrature, J COMPUT APPL MATH 237 (2013) 111-125.

[43] J. Berg, J. Nordström, Superconvergent functional output for timedependent problems using finite differences on summation-by-parts form, J COMPUT PHYS 231 (2012) 6846-6860.

[44] T. Lundquist, J. Nordström, The SBP-SAT technique for initial value problems, J COMPUT PHYS 270 (2014) 86-104.

[45] J. Nordström, A Roadmap to well Posed Problems and Stable Problems in Computational Physics, Accepted in J SCI COMPUT (2016).

[46] B. Gustafsson, H.-O. Kreiss, J. Oliger, Time dependent problems and difference methods, volume 24, John Wiley \& Sons, 1995.

[47] J. Lindström, J. Nordström, A stable and high-order accurate conjugate heat transfer problem, J COMPUT PHYS 229 (2010) 5440-5456.

[48] S. Nikkar, J. Nordström, Fully discrete energy stable high order finite difference methods for hyperbolic problems in deforming domains, J COMPUT PHYS 291 (2015) 82-98. 